\title{
Image Denoising Using a Compressive Sensing Approach Based on Regularization Constraints
}

\author{
Assia El Mahdaoui ${ }^{1}$, Abdeldjalil Ouahabi ${ }^{2, *}$ and Mohamed Said Moulay ${ }^{1}$ \\ 1 Dept. of Analysis, AMNEDP Laboratory, University of Sciences and Technology Houari Boumediene, \\ 16111 Algiers, Algeria; aelmahdaoui@usthb.dz; mmoulay@usthb.dz \\ 2 UMR 1253, iBrain, INSERM, University of Tours, 37000 Tours, France \\ * Correspondence: ouahabi@univ-tours.fr
}

\begin{abstract}
In remote sensing applications, one of the key points is the acquisition, real-time preprocessing and storage of information. Due to the large amount of information present in the form of images or videos, compression of this data is necessary. Compressed sensing (CS) is an efficient technique to meet this challenge. It consists in acquiring a signal, assuming that it can have a sparse representation, using a minimal number of non-adaptive linear measurements. After this CS process, a reconstruction of the original signal must be performed at the receiver. Reconstruction techniques are often unable to preserve the texture of the image and tend to smooth out its details. To overcome this problem, we propose in this work, a CS reconstruction method that combines the total variation regularization and the non-local self-similarity constraint. The optimization of this method is performed by the augmented Lagrangian which avoids the difficult problem of nonlinearity and non-differentiability of the regularization terms. The proposed algorithm, called denoising compressed sensing by regularizations terms (DCSR), will not only perform image reconstruction but also denoising. To evaluate the performance of the proposed algorithm, we compare its performance with state-of-the-art methods, such as Nesterov's algorithm, group-based sparse representation and wavelet-based methods, in terms of denoising, and preservation of edges, texture and image details, as well as from the point of view of computational complexity. Our approach allows to gain up to $25 \%$ in terms of denoising efficiency, and visual quality using two metrics: PSNR and SSIM.
\end{abstract}

Keywords: compressive sensing; image reconstruction; regularization; total variation; augmented Lagrangian; non-local self-similarity; wavelet denoising

\section{Introduction}

Compressed sensing (CS) has already attracted great interest in various fields. Examples include medical imaging [1,2], communication systems [3-6], remote sensing [7], reconstruction algorithm design [8], image storage in databases [9], etc. Compressed sensing provides an alternative approach to Shannon vision to reduce the number of samples, and/or reduce transmission/storage costs. There are other approaches that also address this issue, such as random sampling [10]. Compressed sensing recovery is a linear optimization problem. The most common CS retrieval algorithms explore the prior knowledge that a natural image is sparse in certain domains, such as in the wavelet domain, where simple and efficient noise reduction is possible [11-14], or in the discrete gradient domain that we will develop in this work.

Image recovery in these application domains can be formulated as a linear inverse problem, which can be modeled as follows:

$$
f=A u+\varepsilon
$$

$f \in \mathbb{R}^{M}$ is the observed noisy image, $u \in \mathbb{R}^{N}$ is the unknown clean image, and $\varepsilon$ is an additive noise and $A \in \mathbb{R}^{M \times N}$ is a linear operator. Given $A$, image reconstruction extract $\hat{u}$ 
from $f$ making classical least-squares approximation alone not suitable. To stabilize recovery, regularization techniques frequently used, giving a general reconstruction model of the form:

$$
\underset{u}{\arg \min } \frac{1}{2}\|A u-f\|_{2}^{2}+\lambda \phi_{\text {reg }}(u)
$$

where, $\lambda>0$ is a regularization parameter and \|\|$_{2}$ denotes the $l_{2}$ norm. The fidelity term, $\|A u-f\|_{2}^{2}$, forces the reconstructed image to be close to the original image. The regularization term, $\phi_{\text {reg }}(u)$, actually perform the noise reduction.

Many optimization approaches for regularization based image inverse problems have been developed. Particularly, the total variation (TV) based approach has been one of the most popular and successful approaches; Chambolle [15] introduced the dual approach to the unconstrained real valued case. Later, Beck and Teboulle [16] present a fast computational method based on gradient optimization approach for solving the TVregularized problem. Recently, several methods based on total variation have been proposed for hyperspectral image $[17,18]$ denoising an essential preprocessing step to improve image quality.

However, since the total variation model favors the piecewise constant image structures, the total variation models tend to over smooth the image details, it tends to smooth out the fine details of an image. To overcome these intrinsic draw backs of the total variation model, we introduce a non-local self-similarity constraint, as a complementary regularization into the model. The non-local self-similarity can restore high quality image. To make our algorithm robust, an augmented Lagrangian method is proposed to solve efficiently the above inverse problem.

The remainder of this paper is organized as follows. In section 2, the basics of the methods used to image restoration are presented. The tools of denoising image considered in the proposed framework are described in section 3. Section 4 presents our proposed algorithm, called DCSR. The experimental results from the processing of Lena, Barbara and Cameraman images, available in the public and royalty-free, database https://ccia.ugr.es/cvg/dbimagenes/index.php, and the comparison with competing methods are discussed in Section 5. Finally, conclusions are given in Section 6.

\section{Related Works}

\subsection{Compressive sensing}

The aim of compressive sensing $[19,20]$ is to acquire an unknown signal, assuming to have a sparse representation while using the minimal number of linear non adaptive measurements. After wards, recover it by means of efficient optimization procedures. The three points of compressive sensing theory are the sparsity of the received signals, incoherent measurement matrix and robust algorithm of reconstruction.

Let $x \in \mathbb{R}^{N}$ be a signal having a sparse representation and its sparsity is $K,(K \ll N)$, so that $x$ can be expressed as:

$$
x=\psi u
$$

where, $\psi=\left(\psi_{1}, \psi_{2}, \ldots, \psi_{N}\right)$ is the basis matrix, $u \in \mathbb{R}^{N \times 1}$ the column vector of weighting coefficients with

$$
u_{i}=\left\langle x_{i}, \psi_{i}\right\rangle=\psi^{T} x
$$

using $\phi \in \mathbb{R}^{M \times N}$ as a sensing matrix of dimension $K<M \ll N$. The obtained signal is:

$$
f=\phi x=A u
$$


with $A=\phi \psi^{-1}$. $A$ is an $M \times N$ matrix that verifies the restricted isometry condition of order $K$ :

$$
1-\delta_{K} \leq \frac{\|A u\|_{2}^{2}}{\|u\|_{2}^{2}} \leq 1+\delta_{K}, \quad 0<\delta_{K}<1
$$

The reconstruction of $x$ from $f$ can be obtained via the $l_{1}$ norm minimization as:

$$
\hat{u}=\min \|u\|_{1} \text { subject to } f=A u
$$

More often, when $f$ is contaminated by noise, the equality constraint comes:

$$
\widehat{u}=\min \|u\|_{1} \text { subject to }\|A u-f\|_{2}^{2} \leq \varepsilon
$$

where $\varepsilon>0$ represents a predetermined noise level. For an appropriate scalar weight $\alpha$, we can obtain the following variant of (8):

$$
\min _{u} \frac{1}{2}\|A u-f\|_{2}^{2}+\alpha\|u\|_{1}
$$

Problems (8) and (9) are equivalent in the sense that solving one will determine the parameter in the other such that both give the same solution. If $x$ is sufficiently sparse and the measurement matrix $\psi$ is incoherent with orthogonal basis $\phi$, then $x$ can be reconstructed by solving the $l_{1}$ norm minimization problem.

There are several iterative reconstruction algorithms to solve the problem, such as orthogonal matching pursuit (OMP) [21] and approximate message passing (AMP) [22], an extension of the AMP; denoising based AMP (D-AMP) [23] employs denoising algorithms for CS recovery and can get a high performance for nature images.

\subsection{Augmented Lagrangian}

Augmented Lagrangian method [24] is a new technique that appears with the core idea, which is a combination between the standard Lagrangian function and quadratic penalty function, also known as the method of multipliers.

Considering the following constrained optimization problem:

$$
\min _{u} f(u) \text { subject to } H u=g
$$

where $u \in \mathbb{R}^{N}, g \in \mathbb{R}^{M}$ and $H$ is a matrix of dimension $M \times N$, i.e., there are $M$ linear equality constraints.

The augmented Lagrangian function for this problem is given by:

$$
L(u, \lambda, \rho)=f(u)-\lambda^{\mathrm{T}}(H u-g)+\frac{\rho}{2}\|H u-g\|_{2}^{2}
$$

such as, $\lambda \in \mathbb{R}^{M}$ is a vector of the Lagrange multiplier and $\rho>0$ is the augmented Lagrangian penalty parameter for the quadratic infeasibility term [25]. As described in Algorithm 1, the so-called augmented Lagrangian method consists in finding the global optimal point of the optimization problem, which has two vectors, the Lagrangian multiplier $\lambda$ and the signal $u$, that need to be solved. Through the alternate iteration method to keep one vector fixed and to update the other, the solution can be obtained successfully until the stopping criterion is satisfied. 


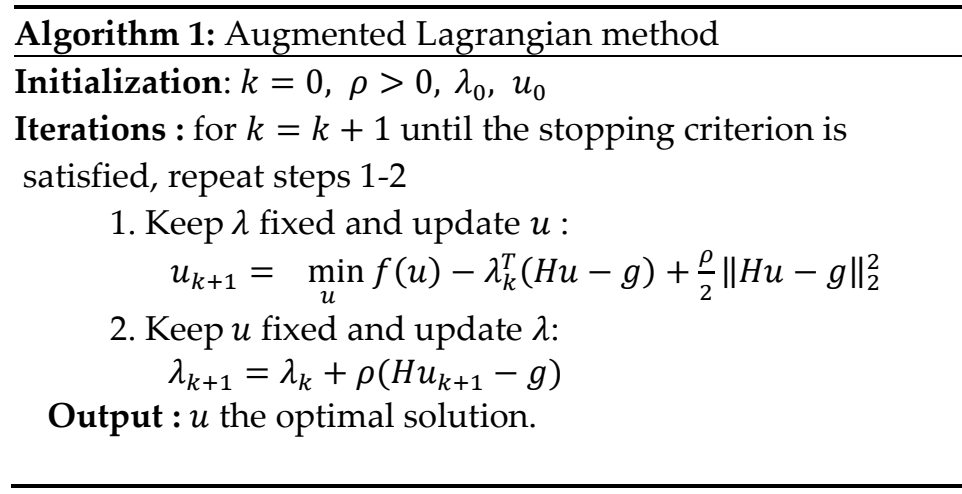

\section{Technical framework for image denoising}

It is well known that existing several methods for image denoising, including total variation image regularization [26], wavelet thresholding [27], non-local means [28], basis pursuit denoising [29], block-matching and 3D filtering [30], among others. These methods can perform image smoothing/denoising as well as preserve edges to a certain extent. If we can obtain more precise measurements of the original images than the corresponding noisy images, then we can reconstruct the image well by CS theory. In particular, we can them into two classes, as special cases of the proposed framework.

\subsection{Regularization functions}

The total variation has been introduced in first by Rudin, Osher and Fatemi [31], as a regularizing criterion for solving inverse problems. Total variation model is a regularization terms demonstrate high effectiveness in preserving edges and recovery smooth regions. The use of total variation regularization in the area of compressive sensing makes the reconstructed images sharper by preserving the edges or boundaries more accurately. Then, the total variation (TV) model can be written as:

$$
T V(u)=\|D u\|_{p}
$$

where $u \in \mathbb{R}^{N}$ represents an image, $D=\left[D_{h}, D_{v}\right]$ and $D_{h}, D_{v}$ denote the horizontal and vertical gradient picture, respectively. The $l_{p}$ norm could either be the $l_{2}$ norm corresponding to the isotropic TV, or the $l_{1}$ norm corresponding to the anisotropic TV. By definition, $l_{p}$ norm is $\|u\|_{p}=\left(\sum_{i=1}^{N}\left|u_{i}\right|^{p}\right)^{\frac{1}{p}}$. In this paper, we consider $p$ equal to 1 .

A non-local self-similarity (NLS) is another significant property of natural images, it was proposed in the first time in the work for image denoising [32]. It depicts the repetitiveness of the textures $[33,34]$ or structures embodied by natural images, which is maintaining properties of initial image (the sharpness and edges) and obtaining in this steps. First, it divides image $u$ size $N$ into many overlapped blocks $u^{i}$ of size $\sqrt{n} \times \sqrt{n}$, at location $i, i=1,2, \ldots, N$. Second, search $(m-1)$ similar blocks and denoted $S_{u^{i}}$ a set including $m$ best block to $u^{i}$ in the training window with $(L \times L)$ size. Third, for each $S_{u^{i}}$ transform the blocks belonging to $S_{u^{i}}$ into 3D array by the superposition denoted by $Z_{u^{i}}$.

$$
N L S(u)=\left\|\theta_{u}\right\|_{1}=\sum_{1}^{N}\left\|T^{3 D}\left(Z_{u^{i}}\right)\right\|_{1}
$$

such that $T^{3 D}$ is the operator of an orthogonal $3 \mathrm{D}$ transform, and $T^{3 D}\left(Z_{u^{i}}\right)$ the transform coefficients for $Z_{u^{i}} . \theta_{u}$ be the column vector of all the transform coefficients of image $u$ with size $K=\sqrt{n} \times \sqrt{n} \times m$ built from all the $T^{3 D}\left(Z_{u^{i}}\right)$ arranged in the lexicographic order. 


\subsection{Wavelet denoising}

Wavelet shrinkage denoising attempts to remove whatever noise present and retain whatever signal is present regardless of the frequency content of the signal. In the wavelet domain, the energy of a natural signal is concentrated in a small number of coefficients; noise is, however, spread over the entire domain. The basic wavelet shrinkage denoising algorithm consist of three steps:

- Discrete wavelet transform (DWT) [35];

- Denoising [36];

- Inverse DWT.

The following is the measurement model:

$$
f=u+\varepsilon
$$

where, $u$ is the original image of size $M \times N$ corrupted by an additive noise $\varepsilon$. The goal is to estimate the denoised image $\hat{u}$ from noisy observation $f$. The elimination of this additive noise can be under the assumption that the appropriate choice of decomposition basis allows discrimination of the useful signal (image) from noise. This hypothesis justifies, in part, the traditional use of denoising by thresholding. There are two thresholding methods frequently used. The soft-thresholding function (also called the shrinkage function):

$$
d_{j}^{\widehat{u}}(k)=\left\{\begin{array}{ccc}
d_{j}^{y}(k)-S & \text { if } & d_{j}^{y}(k)>S \\
d_{j}^{y}(k)+S & \text { if } & d_{j}^{y}(k)<-S \\
0 & & \text { otherwise }
\end{array}\right.
$$

The other popular alternative is the hard-thresholding function:

$$
d_{j}^{\widehat{u}}(k)=\left\{\begin{array}{ccc}
d_{j}^{y}(k) & \text { if } & \left|d_{j}^{y}(k)\right|>S \\
0 & \text { otherwise }
\end{array}\right.
$$

such that, $d_{j}^{y}(k)$ is the wavelet coefficients of the measured signal at level $j$, the estimation of the wavelet coefficients of the useful signal, denoted $d_{j}^{\widehat{u}}(k)$ with the threashold $S=\sigma \sqrt{2 \log N}$, where $N$ is the number of pixels for the test image and $\sigma$ represents the noise standard deviation.

\section{Our efficient image denoising scheme}

In what follows, we substitute the aforementioned results (12) and (13) into (9), we obtain the following problem :

$$
\underset{u}{\arg \min } \frac{1}{2}\|A u-f\|_{2}^{2}+\tau T V(u)+\mu N L S(u)
$$

Lets us recall that $T V(u)=\|D u\|_{1}$ and $N L S(u)=\left\|\theta_{u}\right\|_{1}=\sum_{1}^{N}\left\|T^{3 D}\left(Z_{u^{i}}\right)\right\|_{1}$

The problem can be converted into an equality-constrainted problem by introducing $w$ and $x$,

$$
\min _{w, u, x} \frac{1}{2}\|A u-f\|_{2}^{2}+\tau\|w\|_{1}+\mu\left\|\theta_{x}\right\|_{1} \text { subject to } D u=w, u=x
$$

where $\tau$ and $\mu$ are control parameters. Augmented Lagrangian function of (18) is as follow:

$$
\mathcal{L}_{A}(w, u, x)=\frac{1}{2}\|A u-f\|_{2}^{2}+\tau\|w\|_{1}+\mu\left\|\theta_{x}\right\|_{1}-\gamma^{T}(D u-w)-\varphi^{T}(u-x)+\frac{\mu}{2}\|D u-w\|_{2}^{2}+\frac{\beta}{2}\|u-x\|_{2}^{2}
$$


where $\mu$ and $\beta$ are the penalty parameters corresponding to $\|D u-w\|_{2}^{2}$ and $\|u-x\|_{2}^{2}$ respectively.

To solve (18), we use the augmented Lagrangian method iteratively, as follow:

$$
\left\{\begin{array}{c}
\left(w^{k+1}, u^{k+1}, x^{k+1}\right)=\underset{w, u, x}{\arg \min } \mathcal{L}_{A}(w, u, x) \\
\gamma^{k+1}=\gamma^{k}-\mu\left(D u^{k+1}-w^{k+1}\right) \\
\varphi^{k+1}=\varphi^{k}-\beta\left(u^{k+1}-x^{k+1}\right)
\end{array}\right.
$$

Here, the subscript $k$ denotes the index of the iteration and $\gamma, \varphi$ are the Lagrangian multipliers associated with the constraints $D u=w, u=x$, respectively.

Due to the non differentiability of problem (19), we used the alternative direction method to solve efficiently a problem, which alternatively minimizes one variable while fixing the other variables, to split (19) into the following three sub-problems. For simplicity, the subscript $k$ is omitted without confusion.

- Update $w$

Given $u$ and $x$, the optimization problem associated with $w$ as follow:

$$
\underset{w}{\arg \min } \tau\|w\|_{1}-\gamma^{T}(D u-w)+\frac{\mu}{2}\|D u-w\|_{2}^{2}
$$

according to [37], the solution of $\mathrm{w}$ sub-problem is written as:

$$
\widetilde{w}=\max \left\{\left|D u-\frac{\gamma}{\mu}\right|-\frac{\tau}{\mu}, 0\right\} \operatorname{sgn}\left(D u-\frac{\gamma}{\mu}\right)
$$

where $\max \{\cdot\}$ represents the larger number between two elements, and $\operatorname{sgn}(\cdot)$ is a piecewise function defined as follows:

$$
\operatorname{sgn}(x)=\left\{\begin{array}{cl}
-1 & \text { if } x<0 \\
0 & \text { if } x=0 \\
1 & \text { if } x>0
\end{array}\right.
$$

- Update u

Given $w$ and $x$, the sub-problem associated with $u$ is equivalent to

$$
\underset{u}{\arg \min } \frac{1}{2}\|A u-f\|_{2}^{2}-\gamma^{T}(D u-w)-\varphi^{T}(u-x)+\frac{\mu}{2}\|D u-w\|_{2}^{2}+\frac{\beta}{2}\|u-x\|_{2}^{2}
$$

which simplifies to the linear system

$$
\left(D^{T} D+\left(\frac{\beta}{\mu}+\frac{1}{\mu}\right) I\right) u=\frac{1}{\mu} A^{T} f+D^{T}\left(w-\frac{\gamma}{\mu}\right)+\left(\frac{\beta}{\mu} x-\frac{\varphi}{\mu}\right)
$$

We observe that $D^{T} D$ is a positive semi definite tridiagonal matrix. Since $\mu$ and $\beta$ are both positive scalars, the matrix on the left-hand-side of the above system is positive definite tridiagonal.

- Update $x$

Given $w$ and $u$, the $x$ sub-problem becomes

$$
\underset{x}{\arg \min } \mu\left\|\theta_{x}\right\|_{1}+\frac{\beta}{2}\|u-x\|_{2}^{2}-\varphi^{T}(u-x)
$$

the problem (25) can be reformulated below: 


$$
\underset{x}{\arg \min } \frac{1}{2}\|x-r\|_{2}^{2}+\frac{\mu}{\beta}\left\|\theta_{x}\right\|_{1}
$$

Considering $r=u-c$ with $c=\frac{\varphi}{\beta}$, as a noisy observation of $x$, the error (or noise) $e=x-$ $r$ follows a probability law, not necessarily Gaussian, with zero mean and variance $\sigma^{2}$. According to the central limit theorem or law of large numbers, the following equation holds:

$$
\frac{1}{N}\|x-r\|_{2}^{2}=\frac{1}{K}\left\|\theta_{x}-\theta_{r}\right\|_{2}^{2}
$$

with $e, x, r \in \mathbb{R}^{N}$ and $\theta_{x}, \theta_{r} \in \mathbb{R}^{i}$ for $i=1, \ldots, N$

equivalent to

$$
\|x-r\|_{2}^{2}=\frac{N}{K}\left\|\theta_{x}-\theta_{r}\right\|_{2}^{2}
$$

incorporating (28) into (26) leads to

$$
\underset{x}{\arg \min } \frac{1}{2}\left\|\theta_{x}-\theta_{r}\right\|_{2}^{2}+\frac{K \mu}{N \beta}\left\|\theta_{x}\right\|_{1}
$$

since the unknown variable $\theta_{x}$ is component-wise separable in (29), such that

$$
\begin{gathered}
\widehat{\theta_{x}}=\operatorname{soft}\left(\theta_{r}, \sqrt{2 \varphi}\right), \quad \varphi=\frac{K \mu}{N \beta}, \quad K=\sqrt{n} \times \sqrt{n} \times m \\
\widehat{\theta_{x}}=\operatorname{sgn}\left(\theta_{r}\right) \max \left\{\left|\theta_{r}\right|-\sqrt{2 \varphi}, 0\right\}
\end{gathered}
$$

Thus, the closest solution from $x$ sub-problem (25) is follow:

$$
\tilde{x}=\Omega\left(\widehat{\theta_{x}}\right)
$$

where $\Omega$ is the reconstruction operator.

Based on the discussions above, we get the algorithm for solving (17) shown below:

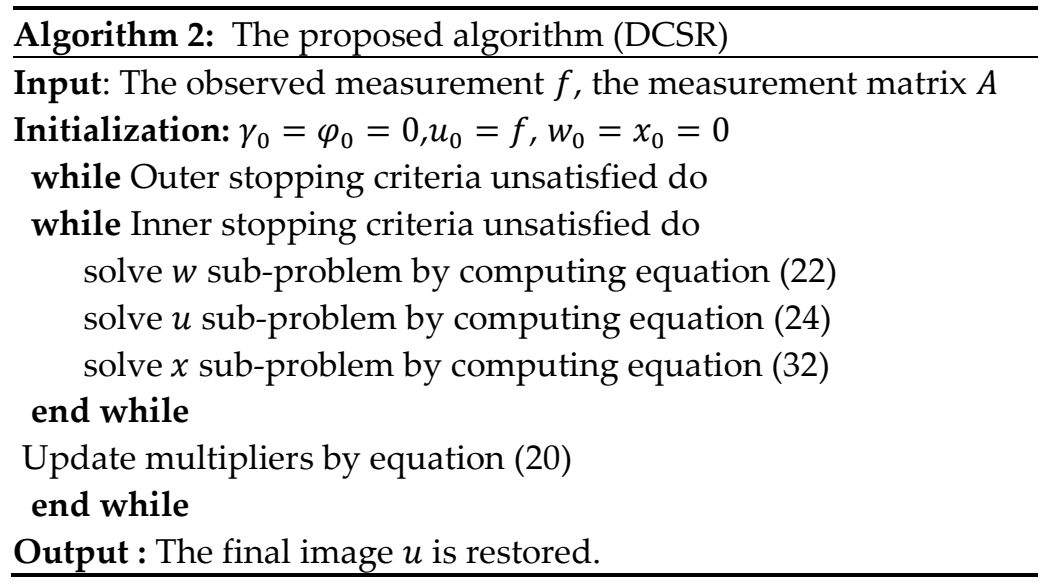

The algorithm detailed above is used to recover corrupted images by white Gaussian noise and salt pepper noise.

Comparative performance of competing methods to recovery noisy images is discussed in the next section. 


\section{Experimental results and discussion}

In this section, we describe the experiments undertaken with both simulated and real data sets to verify the efficiency and practicability of the proposed method. To evaluate the performance of the proposed algorithm, DCSR, we compare it with three other popular CS recovery algorithms: The first algorithm, wavelet thresholding, denoising natural images by assuming they are sparse in the wavelet domain. It transforms signals into a wavelet basis, thresholds the coefficients, and then inverses the transform. The second algorithm, NESTA is based on Nesterov's smoothing technique, extended to TV minimization by modifying the smooth approximation of the objective function. The third algorithm, group-based sparse representation (GSR) enforces image sparsity and selfsimilarity simultaneously under a unified framework in an adaptive group domain.

We used three images, Barbara and Cameraman gray-scale images with size of $256 \times 256$ and color Lena image $512 \times 512$ in our experiments, see Figure 1 .

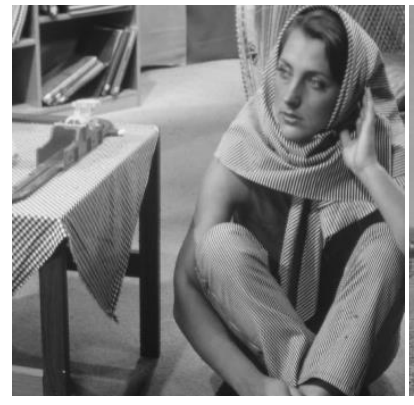

(a)

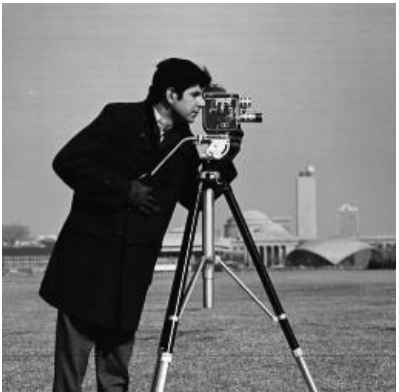

(b)

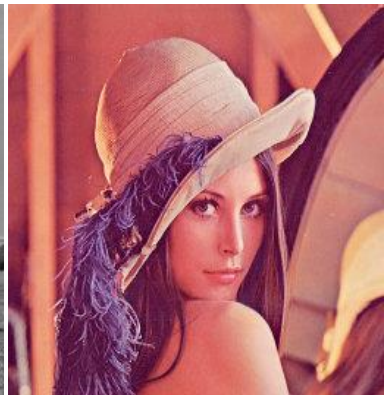

(c)

Figure 1. Test images used in the experiments. (a) Barbara, (b) Cameraman, (c) Lena.

In the simulations, two types of noise were used: the additive white Gaussian noise (AWGN) measured by its standard deviation $\sigma$ and its mean $m$ and the salt and pepper noise.

The salt and pepper noise on image has the following form:

$$
\eta(p)=\left\{\begin{array}{cl}
v_{\max } & \text { with probability } q_{1} \\
v_{\min } & \text { with probability } q_{2}
\end{array}\right.
$$

where $p=\left(p_{1}, p_{2}, \ldots, p_{n}\right) \in \mathbb{R}^{n}$ pixels and $v_{\max }$ is the pixel intensity of salt pixels and $v_{\min }$ is the pixel intensity of pepper pixels. The sum $q=q_{1}+q_{2}$ is the level of the salt and pepper noise.

\subsection{Visual Quality Comparison}

First, we evaluate the performance of our DCSR algorithm by performing experiments on Barbara image corrupted by white Gaussian noise with standard deviation $\sigma$ ranging from 20 to 80 . Figure 2 shows the recovery image results with different values of noises $\sigma$. 


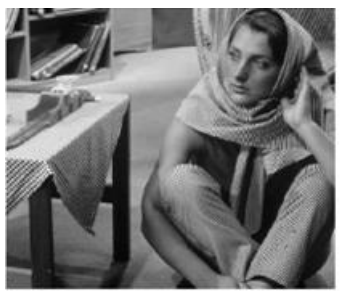

(a)

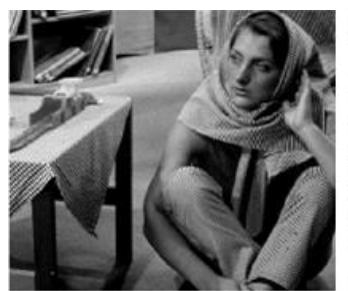

(b)

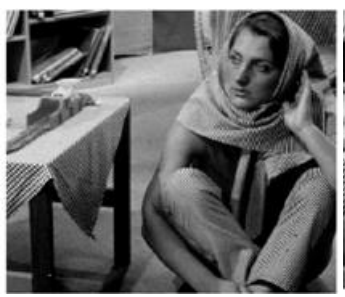

(c)

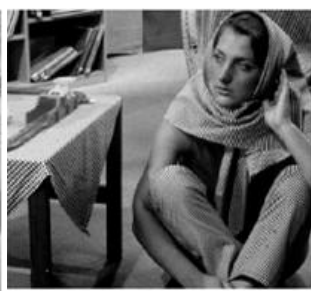

(d)

Figure 2. Visual comparison of the reconstruction quality of the proposed DCSR algorithm for different noise levels. (a) $\sigma=20$, (b) $\sigma=50$, (c) $\sigma=60$, (d) $\sigma=80$.

From Figure 2, it can be seen that the details of Barbara image are preserved very well after denoising with noise from 20 to 80.

The pleasant visual quality is important for a viewer. In order to verify the superiority of the proposed method, we compare it with GSR algorithm, wavelet denoising and NESTA algorithm. We obtain a recovered image from each solver. Comparison experiments are shown in Figures 3, 4 and 5.

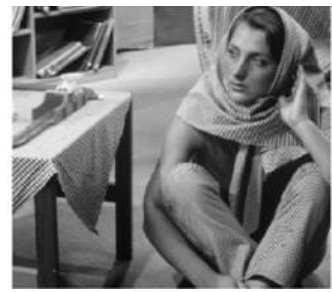

(a)

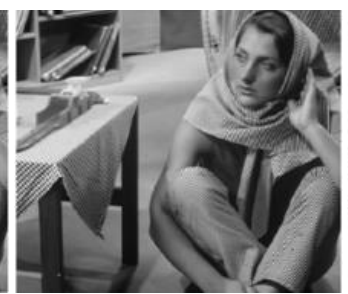

(b)

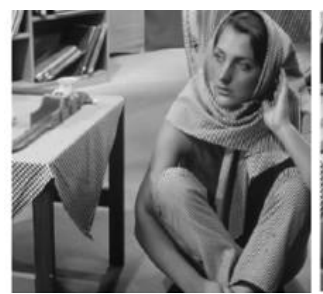

(c)

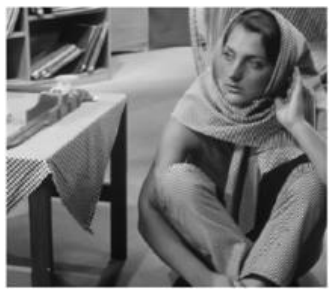

(d)

Figure 3. Visual comparison of the reconstruction quality of GSR algorithm for different noise levels. (a) $\sigma=20$, (b) $\sigma=50$, (c) $\sigma=60$, (d) $\sigma=80$.

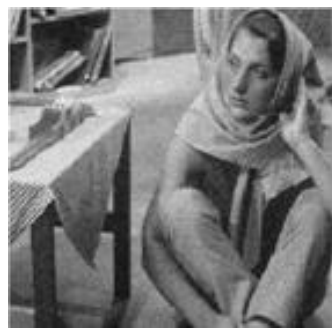

(a)

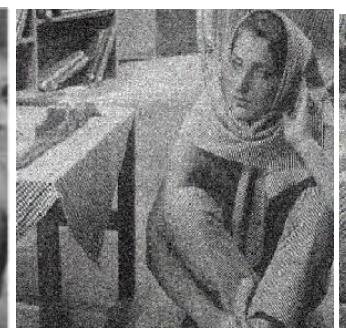

(b)

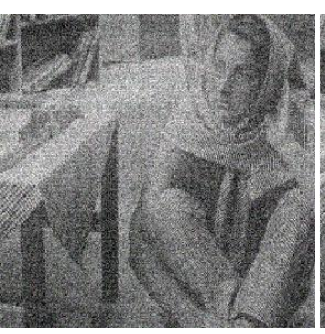

(c)

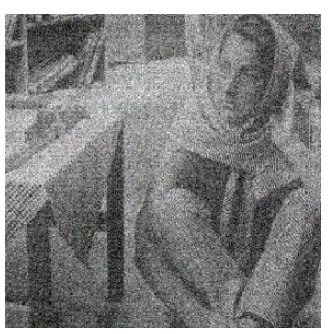

(d)

Figure 4. Visual comparison of the reconstruction quality of the wavelet denoising algorithm for different noise levels. (a) $\sigma=20$, (b) $\sigma=50$, (c) $\sigma=60$, (d) $\sigma=80$.

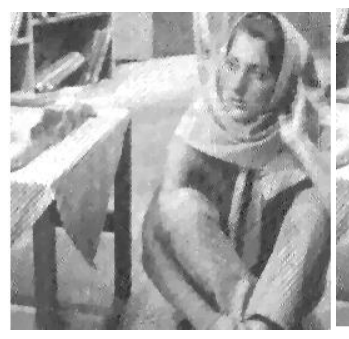

(a)

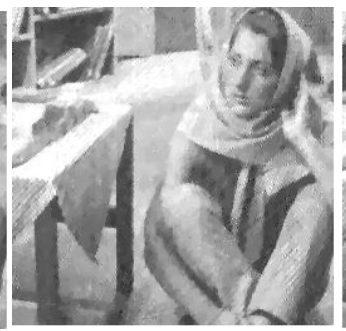

(b)

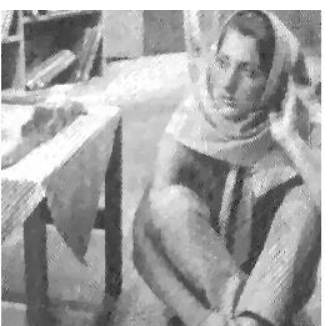

(c)

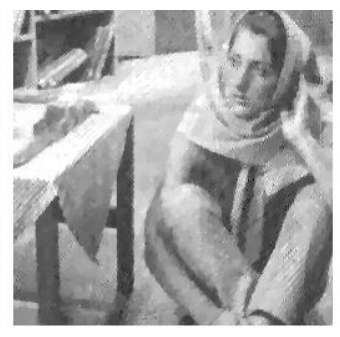

(d)

Figure 5. Visual comparison of the reconstruction quality of NESTA algorithm for different noise levels. (a) $\sigma=20$, (b) $\sigma=50$, (c) $\sigma=60$, (d) $\sigma=80$. 
This image Barbara is relatively complex with rich texture and geometry structure. The images reconstructed by NESTA algorithm are visually unpleasant and lose some important details, for wavelet denoising if the standard deviation $\sigma$ is set too large (greater than 50) the image noise can not be suppressed. Also, one can see that GSR is effective in suppressing the noises. By comparing, the proposed method provides the most visually pleasant results in both edges and textures.

Secondly, we handle the proposed approach to process the impulsive noise salt and pepper in various noise levels varying from $20 \%$ to $70 \%$ and compared its denoising performance with several denoising algorithms: GSR algorithm, NESTA algorithm and wavelet denoising. Figure 6 provides visual results for different algorithm and several noise levels, it can be found that the image Cameraman has been reconstructed very well by our algorithm, DCSR, even with high noise level greater than 50\%. We can conclude that DCSR method presents the best quality image compared to other methods.

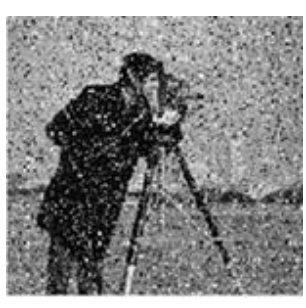

Noise level $20 \%$

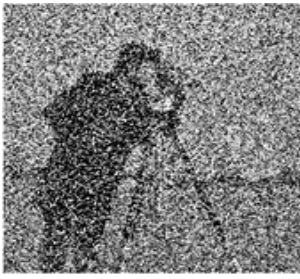

Noise level 50\%

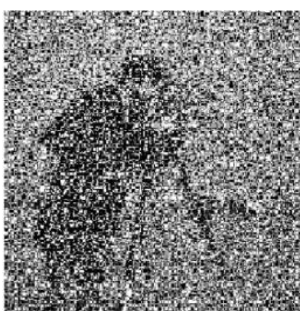

Noise level $60 \%$

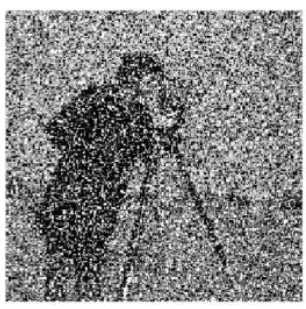

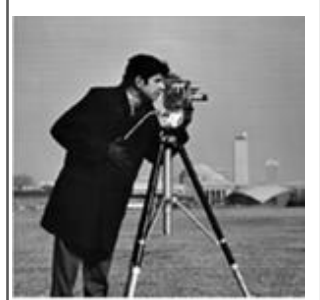
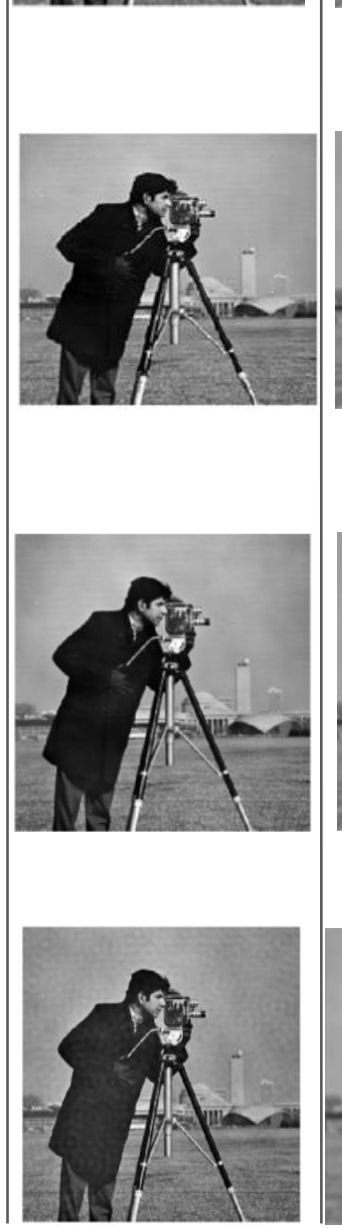
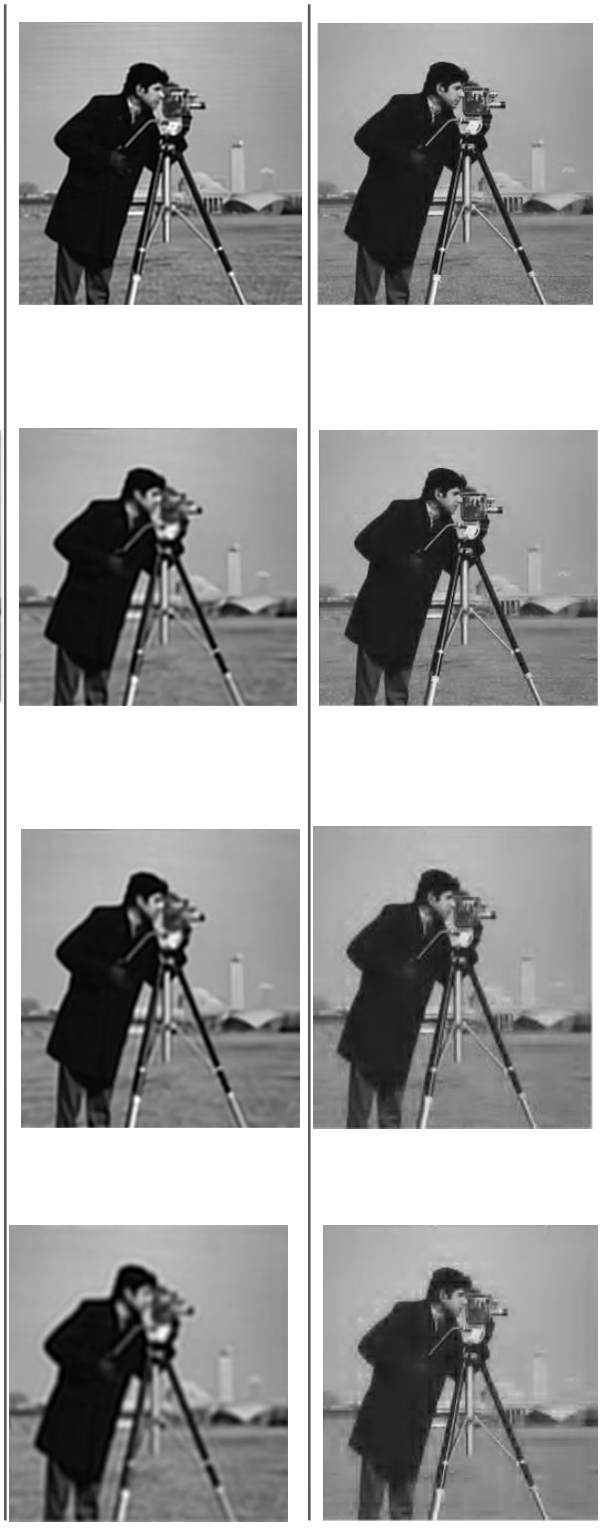
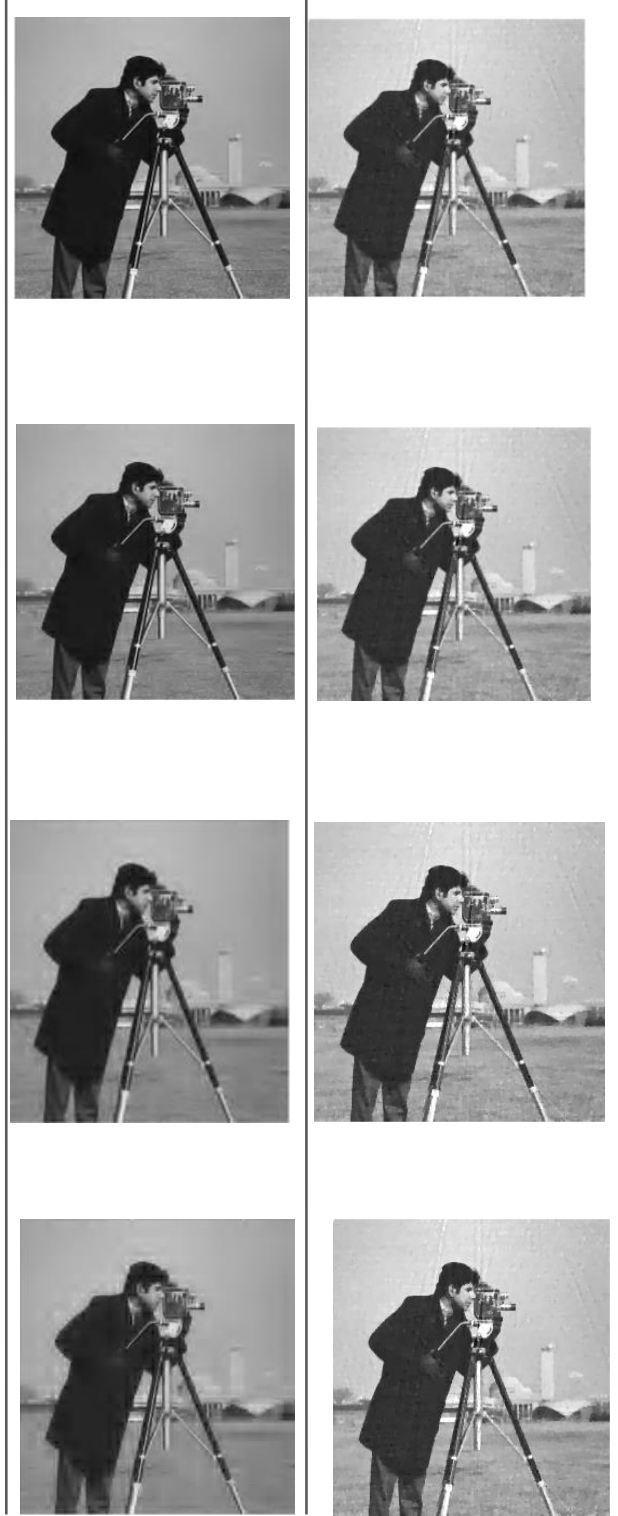

Noise level 70\%

Figure 6. Visual comparison of the reconstruction quality for different noise levels in the case of salt and pepper (see column 1 located on the left side). The algorithms used are, DCSR, GSR, wavelet denoising and NESTA: The denoised images are arranged in columns 2, 3, 4 and 5 respectively. 


\subsection{Image Quality Metrics}

The image processing community has long been using PSNR and MSE as fidelity metrics. The formulas are simple to understand and implement; they are easy and fast to compute and minimizing MSE is also very well understood from a mathematical point of view.

PSNR (peak signal-to-noise ratio, unit: $\mathrm{dB}$ ) [38] is the ratio between the maximum possible power of a signal and the power of noise. The higher PSNR value means the better visual quality. PSNR is defined as follows:

$$
\begin{gathered}
\text { PSNR }=10 \log _{10} \frac{\left(2^{k}-1\right)^{2}}{\mathrm{MSE}} \\
\text { MSE }=\frac{1}{M N} \sum_{i=1}^{M} \sum_{j=1}^{N}(u(i, j)-\hat{u}(i, j))^{2}
\end{gathered}
$$

MSE represents the mean square error between two images, $u$ is the initial image and $\hat{u}$ is the reconstructed image of size $M \times N, i$ and $j$ represent the image row and column pixel position respectively, $k$ is the number of bits of each sample value.

As an alternative to data metrics described above, better visual quality measures have been designed taking into account the effects of distortions on perceived quality. Note that, structural similarity (SSIM) textures [39] is a proven to be a better error metric for comparing the image quality and it is in the range $[0,1]$ with value closer to one indicating better structure preservation.

$$
\operatorname{SSIM}=l(u, \hat{u}) \times c(u, \hat{u}) \times s(u, \hat{u})
$$

such that, $l(i, j)$ is the luminance comparison defined as a function of the mean intensities $\mu_{i}$ and $\mu_{j}$ of signals $i$ and $j$ :

$$
l(i, j)=\frac{2 \mu_{i} \mu_{j}+c_{1}}{\mu_{i}^{2}+\mu_{j}^{2}+c_{1}}
$$

The contrast comparison $c(i, j)$ is a function of standard deviations $\sigma_{i}$ and $\sigma_{j}$, and takes the following form:

$$
c(i, j)=\frac{2 \sigma_{i} \sigma_{j}+c_{2}}{\sigma_{i}^{2}+\sigma_{j}^{2}+c_{2}}
$$

The structure comparison $s(i, j)$ is defined as follows:

$$
s(i, j)=\frac{\sigma_{i j}+c_{3}}{\sigma_{i} \sigma_{j}+c_{3}}
$$

where, $\mu_{i}$ and $\mu_{j}$ represent the mean value of images $u$ and $\hat{u}$ respectively. $\sigma_{i}^{2}$ and $\sigma_{j}^{2}$ represent the variance of images $u$ and $\hat{u}$ respectively. $\sigma_{i j}$ is the covariance of images $u$ and $\hat{u} . c_{1}, c_{2}$ and $c_{3}$ are constants.

Specifically, it is possible to choose $c_{i}=K_{i}^{2} D^{2}, i=1$ and $K_{i}$ is a constant, such as $K_{i} \ll 1$ and $D$ is the dynamic range of the pixel values $(D=255$ corresponds to a grey-scale digital image when the number of bits/pixel is 8 ). 


\subsection{Quantitative assessment}

In this sub-section, we evaluate the quality of image reconstruction. We compare these methods furthermore quantitatively; the peak signal to noise ratio (PSNR) and structural similarity (SSIM) indices are calculated for the images with different algorithms.

Remember that, at first gray image Barbara was contaminated by Gaussian white noise with different values of standard deviation $\sigma$ and denoising with different algorithms. The results of the PSNR values by various algorithms are shown in Figures 710. Let us recall that, the higher PSNR indicates superior image quality and a good performance of algorithm. The values of PSNR illustrate that, obviously, DCSR yields a higher PSNR than the other three methods.

PSNR: $38.75 \mathrm{~dB}$

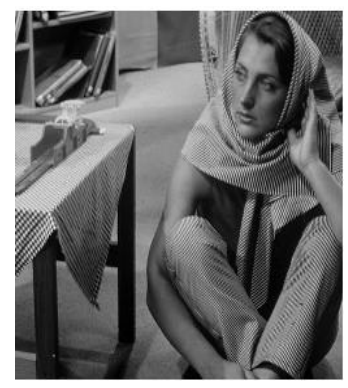

PSNR: $35.16 \mathrm{~dB}$

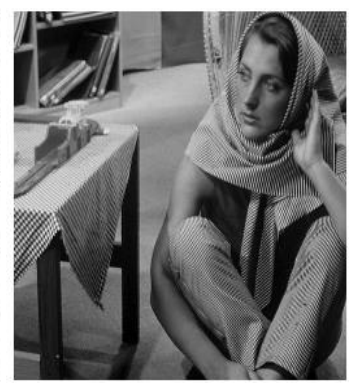

PSNR: $34.67 \mathrm{~dB}$

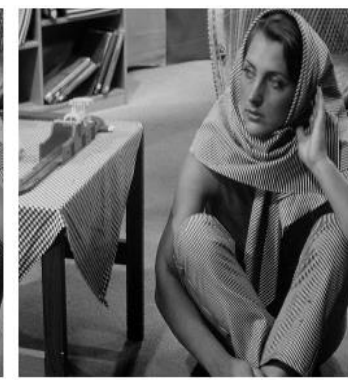

PSNR: $33.59 \mathrm{~dB}$

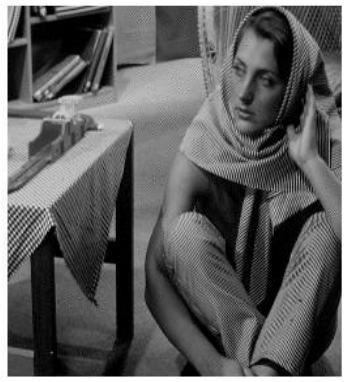

Figure 7. PSNR for different noise levels using the proposed DCSR. From left to right $\sigma=20,50$, 60 and 80 respectively.

PSNR: $34.07 \mathrm{~dB}$

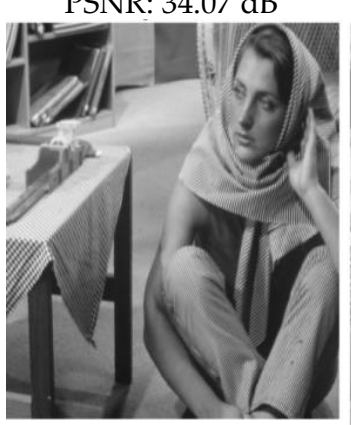

PSNR: $29.10 \mathrm{~dB}$

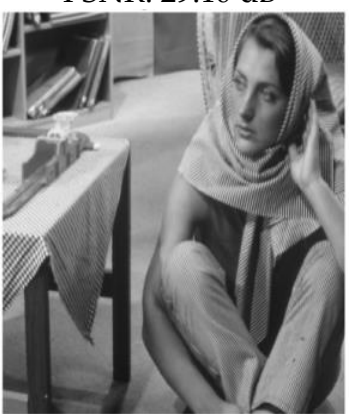

PSNR: $27.39 \mathrm{~dB}$

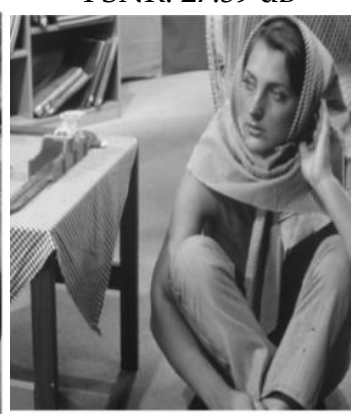

PSNR: $24.49 \mathrm{~dB}$

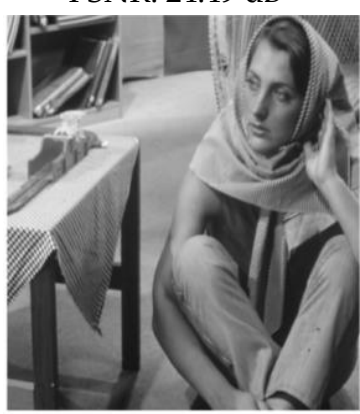

Figure 8. PSNR for different noise levels using GSR algorithm. From left to right $\sigma=20,50,60$ and 80 respectively.
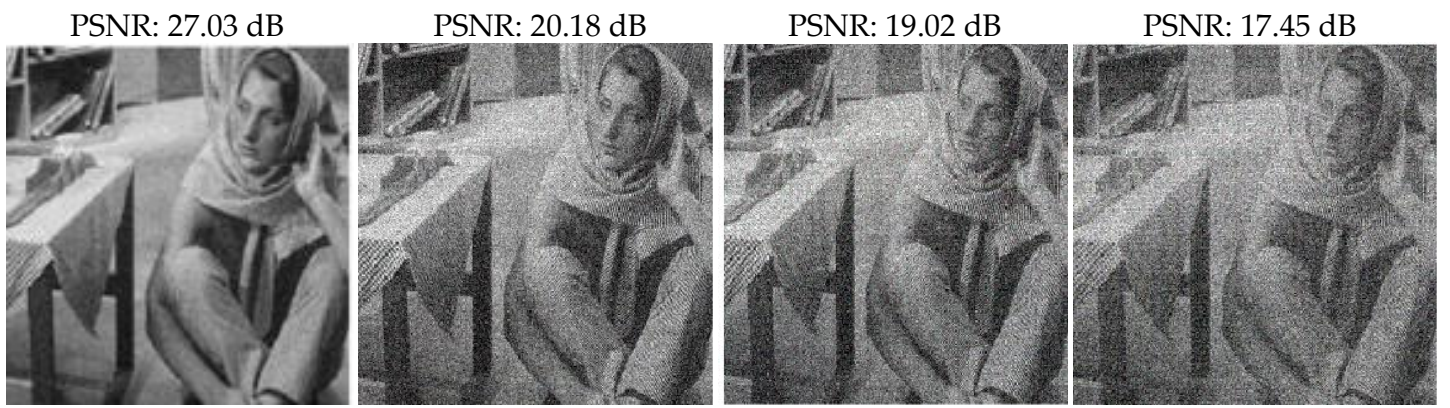

Figure 9. PSNR for different noise levels using wavelet denoising. From left to right $\sigma=20,50,60$ and 80 respectively. 
PSNR: $19.15 \mathrm{~dB}$

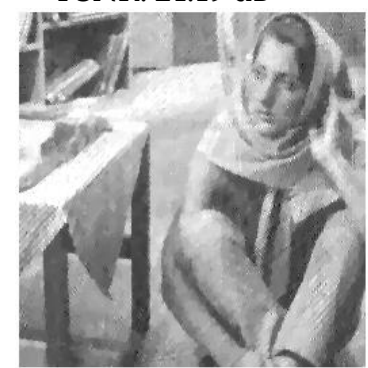

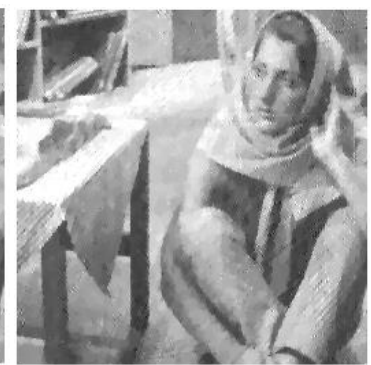

PSNR: $18.08 \mathrm{~dB}$

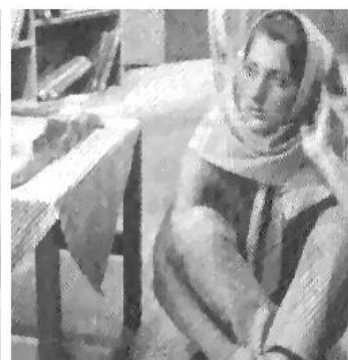

PSNR: $15.46 \mathrm{~dB}$

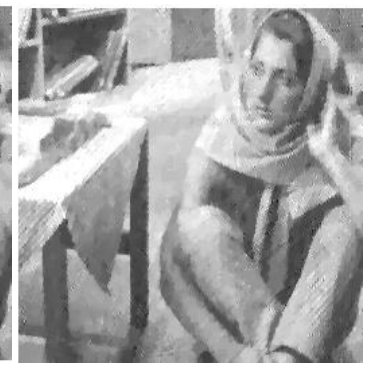

Figure 10. PSNR for different noise levels using NESTA algorithm. From left to right $\sigma=20,50$, 60 and 80 respectively.

Figure 11 presents the performance analysis of four denoising methods for grayscale image corrupted by additive white Gaussian noise. We can clearly see that DCSR our algorithm exhibits the best performance with high PSNR (low noise level) and also with low PSNR (high noise level).

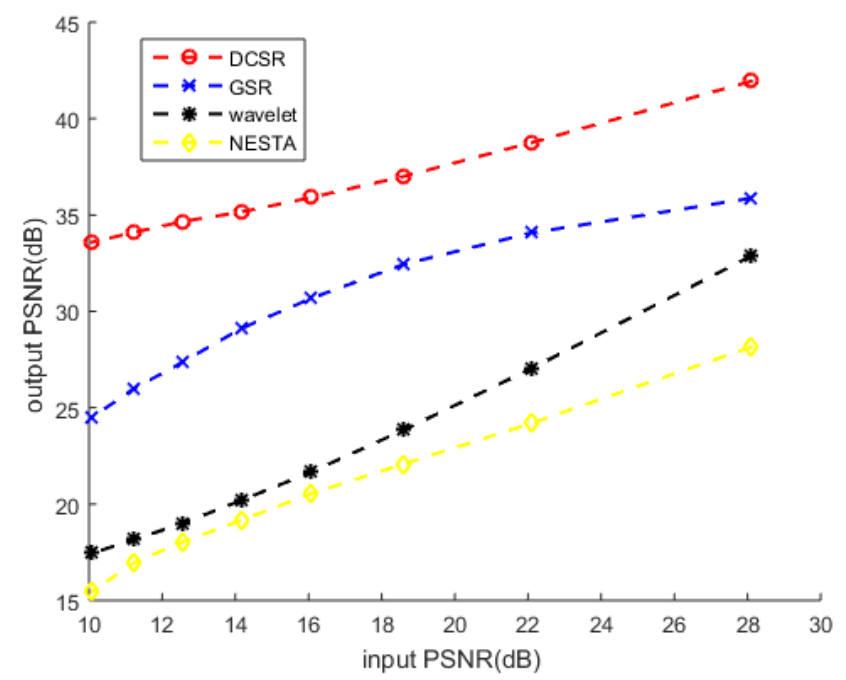

Figure 11. Grayscale image corrupted by additive white Gaussian noise (AWGN): Performance analysis of four denoising methods.

To quantitatively evaluate the effectiveness of our reconstruction-de-noising method, the test image used is corrupted by salt and pepper noise with different noise levels.. Table 1 shows the quantitative assessment results PSNR and SSIM of our algorithm, DCSR, for several values of the noise levels, as well as results obtained using GSR, wavelet and NESTA. 
Table 1. Quality metrics results on different algorithms for different values of noise levels.

\begin{tabular}{cccc}
\hline Method & Noise level & PSNR & SSIM \\
\hline \multirow{3}{*}{ Ours (DCSR) } & $20 \%$ & $\mathbf{3 3 . 9 7}$ & $\mathbf{0 . 9 9}$ \\
& $50 \%$ & $\mathbf{3 0 . 0 8}$ & $\mathbf{0 . 9 5}$ \\
& $60 \%$ & $\mathbf{2 7 . 0 3}$ & $\mathbf{0 . 9 3}$ \\
& $70 \%$ & $\mathbf{2 5 . 2 4}$ & $\mathbf{0 . 9 2}$ \\
\hline \multirow{3}{*}{ GSR algorithm } & $20 \%$ & 26.70 & 0.80 \\
& $50 \%$ & 24.66 & 0.78 \\
& $60 \%$ & 23.61 & 0.74 \\
Wavelet denoising & $70 \%$ & 22.40 & 0.69 \\
\hline & $20 \%$ & 31.68 & 0.89 \\
& $50 \%$ & 28.61 & 0.86 \\
& $60 \%$ & 26.69 & 0.79 \\
& $70 \%$ & 24.84 & 0.74 \\
\hline \multirow{3}{*}{ NESTA algorithm } & $20 \%$ & 18.88 & 0.39 \\
& $50 \%$ & 16.71 & 0.38 \\
& $60 \%$ & 16.33 & 0.37 \\
\hline
\end{tabular}

The best results are highlighted in bold type font. Table 1 indicates that, the PSNR and SSIM values obtained by NESTA are lower, indicating that there are a limitation on the restoration images although being a successful image recover method. GSR and wavelet obtain intermediate PSNR values and SSIM values. Table 1 validates that our method has superiority in image reconstruction compared with the three methods.

This performance is confirmed by Figure 12 which illustrates the PSNR output variations with respect to the input PSNR.

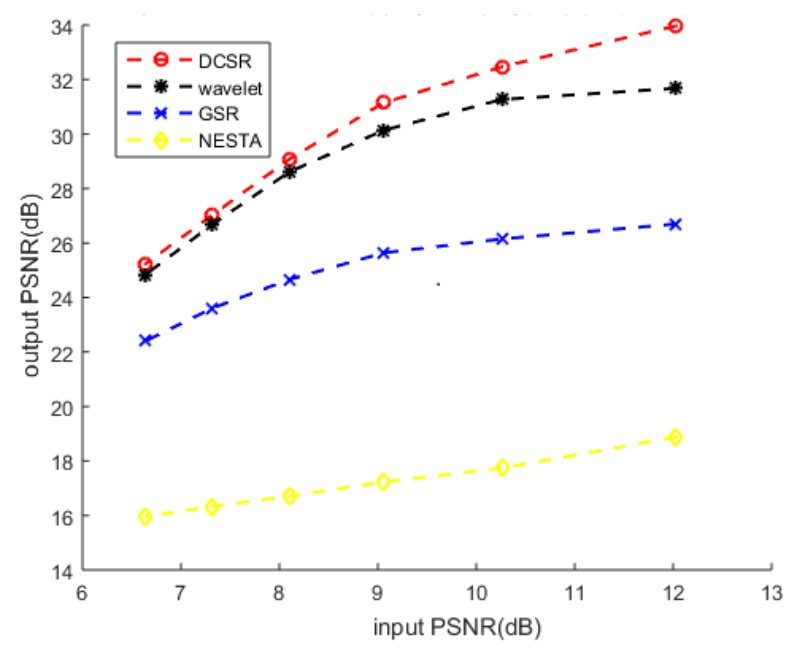

Figure 12. Grayscale image corrupted by salt and pepper noise: Performance analysis of four denoising methods.

The next challenge is to apply this DCSR algorithm to color, or multidimensional, images. This is esential since most digital images used in the modern world are not grayscale, but usually operate in either the RGB or YCbCr color spaces. Both these colors paces are 3-dimensional.

We evaluate the proposed DCSR algorithm on color image Lena. Lena is a good test image because it has a nice mixture of detail, flat regions, shading area and texture. We mainly compare our proposed method to wavelet denoising and GSR algorithm. 
As mentioned previously, two types of noise are used in these experiments: AWGN with a standard deviation $\sigma$ and several salt and pepper noise levels.

At the beginning, additive white Gaussian noise with different values of standard deviation $\sigma=20,50,60$ and 80 are added to this testing image to generate the noisy observations.

Denoising results of this image with different algorithm are shown in Figure 13. From Figure, the proposed method achieves the highest scores of PSNR in all the cases, which fully demonstrates that the denoising results by the proposed method are the best both objectively and visually.

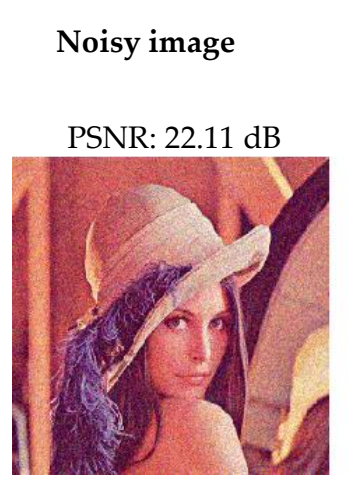

$\boldsymbol{\sigma}=20$

PSNR: $14.17 \mathrm{~dB}$

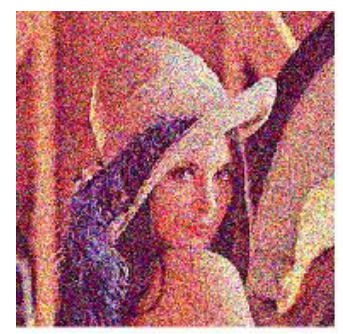

$\boldsymbol{\sigma}=\mathbf{5 0}$

\section{PSNR: $12.57 \mathrm{~dB}$}

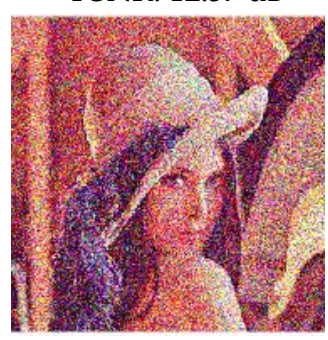

$\sigma=60$

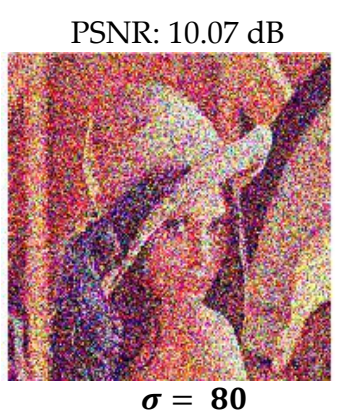

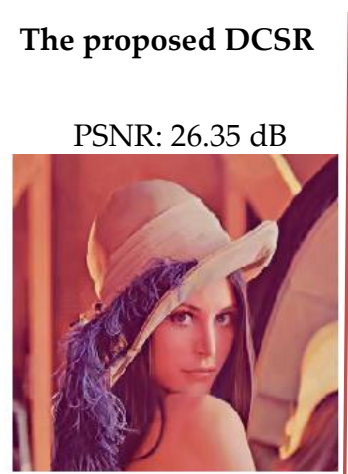
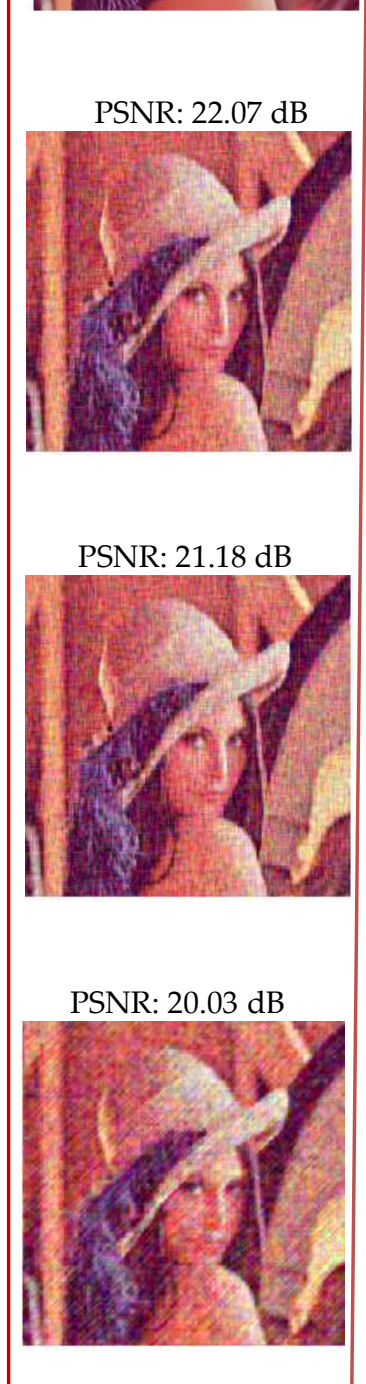

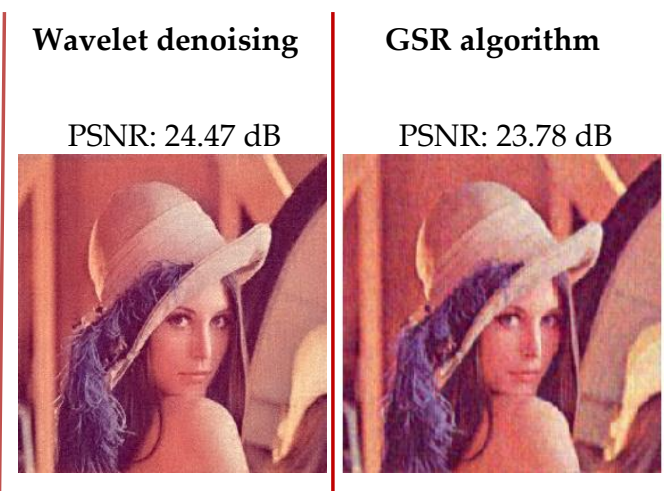

PSN: $20.19 \mathrm{~dB}$
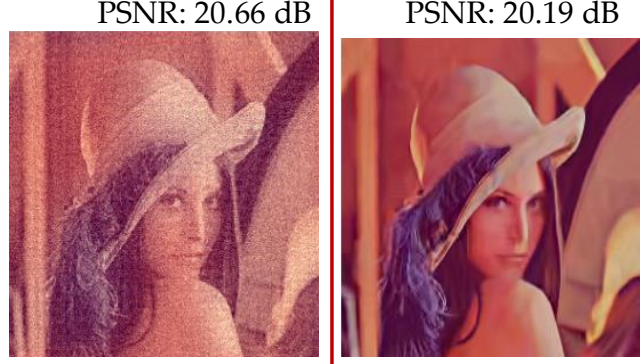

PSNR: $19.75 \mathrm{~dB}$

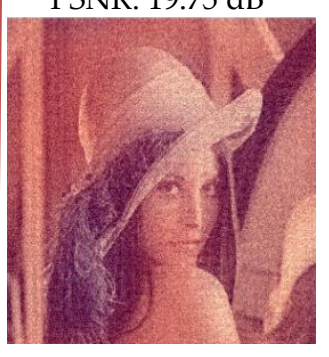

PSNR: $19.56 \mathrm{~dB}$
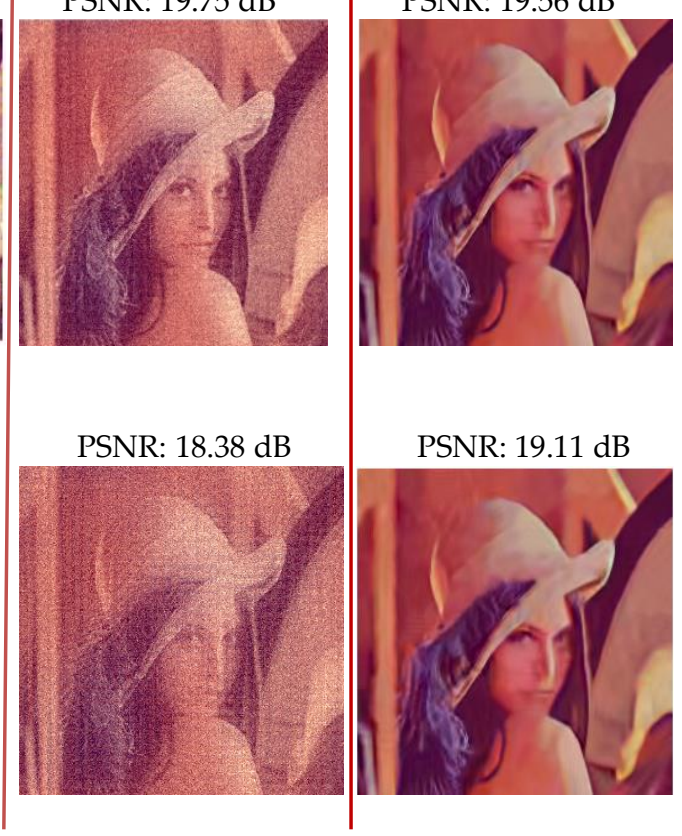

PSNR: $19.11 \mathrm{~dB}$

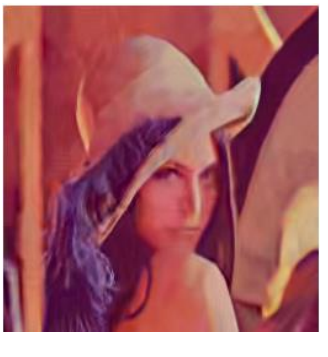

Figure 13. Restoration results of the AWGN-corrupted Lena image for different values of $\sigma$ (see column 1 located on the left side of the figure). The algorithms used, the proposed DCSR, wavelet denoising and GSR, are arranged in columns 2, 3 and 4 respectively. 


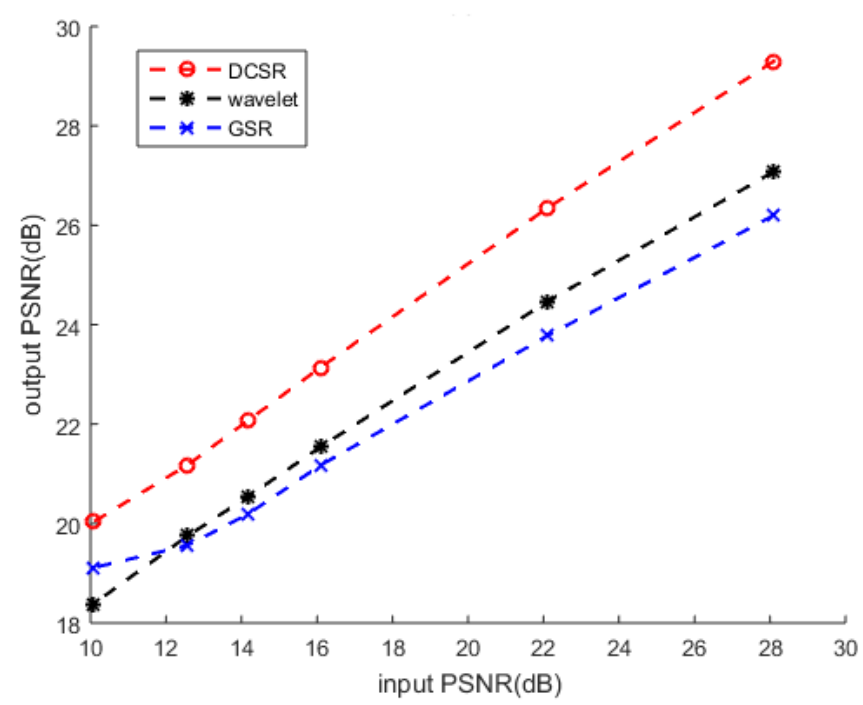

Figure 14. Color image corrupted by AWGN: Performance analysis of three denoising methods.

Figure 14 illustrates the output results evaluated at different noise levels. DCSR algorithm presents good denoising performance with distinctive results in presence of high noise levels.

In this second step, we added the salt and pepper noise with different noise level 10\%, $15 \%, 20 \%$ and $30 \%$ to Lena to obtain the noised observations, respectively. Then, we apply our denoise algorithm to restore the noisy images and compared it with the others two algorithms: wavelet denoising and GSR algorithm. Figure 15 indicates that DCSR achieves results with better visual quality. This performance is confirmed by Figure 16 which illustrates the PSNR variations with respect to the input PSNR. The robustness of our algorithm towards the noise level is verified to a certain level.

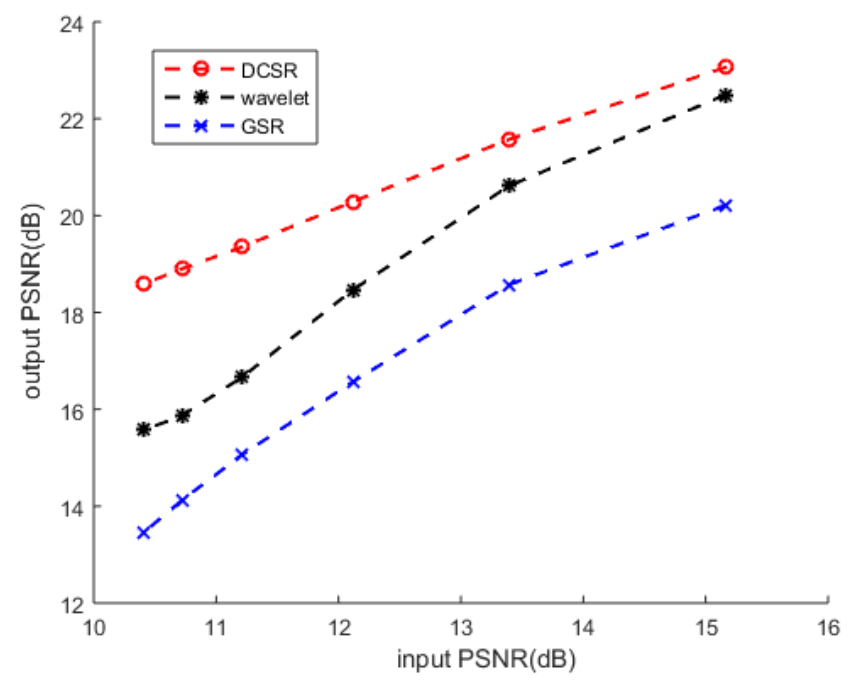

Figure 16. Color image corrupted by salt and pepper noise: Performance analysis of three denoising methods.

From these results, the proposed method achieves the highest scores of PSNR and SSIM in all the cases, which fully demonstrates that the restoration results by the proposed method are the best both objectively and visually 


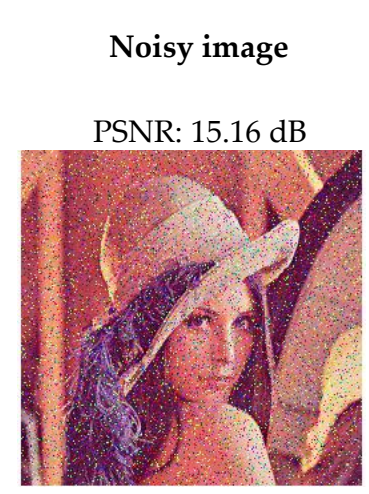

Noise level $10 \%$

PSNR: $13.39 \mathrm{~dB}$

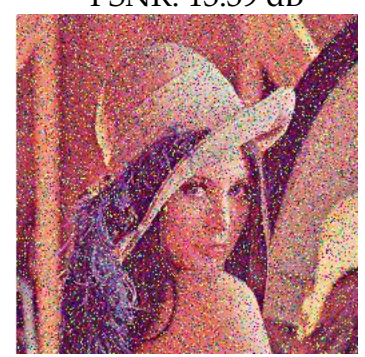

Noise level 15\%

PSNR: $12.21 \mathrm{~dB}$

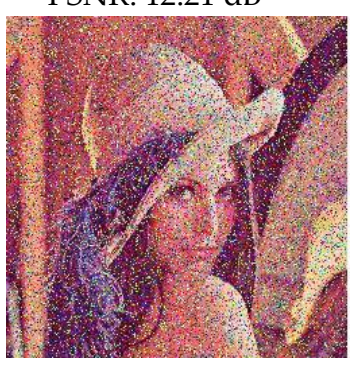

Noise level $20 \%$
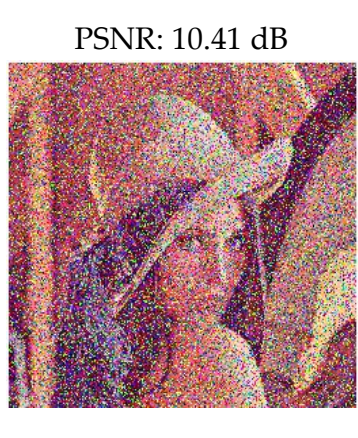

Noise level $30 \%$
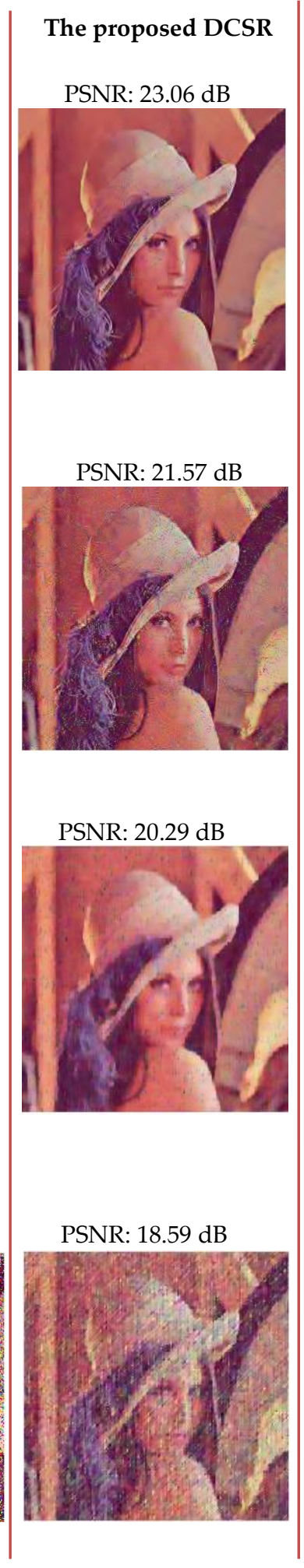
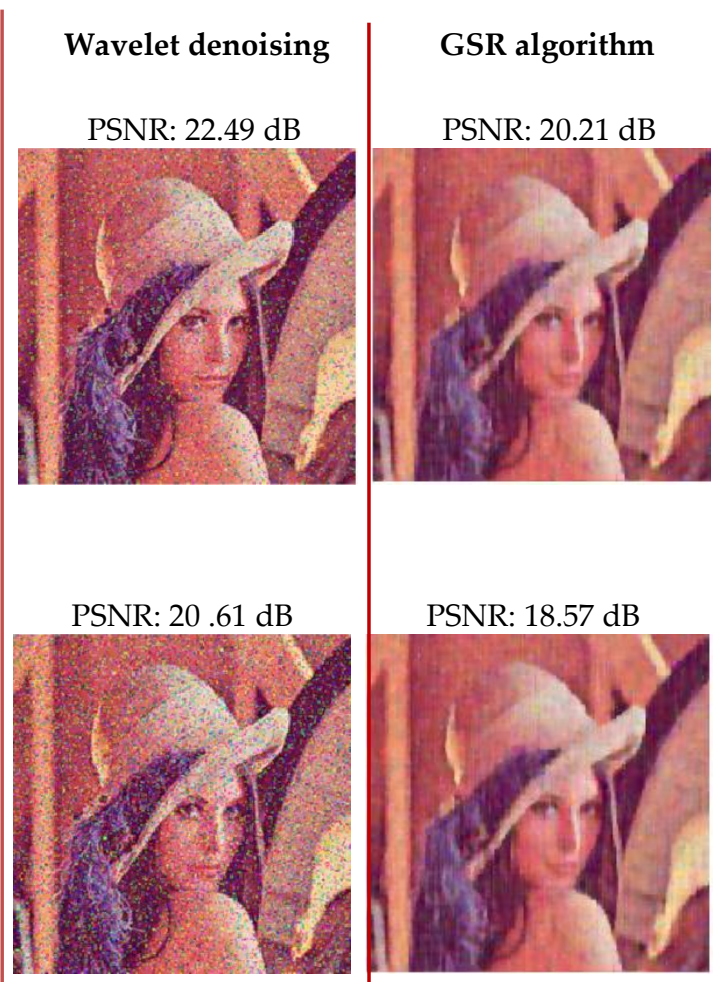

PSNR: $18.57 \mathrm{~dB}$

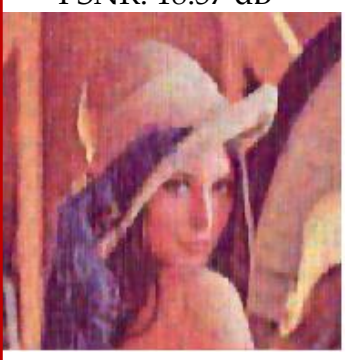

PSNR: $18.47 \mathrm{~dB}$

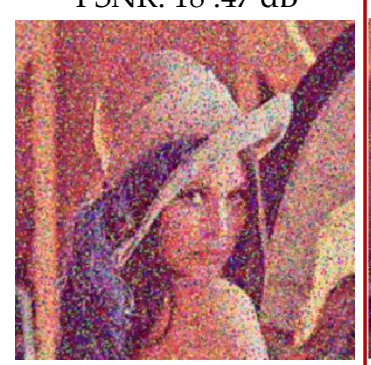

PSNR: $16.57 \mathrm{~dB}$
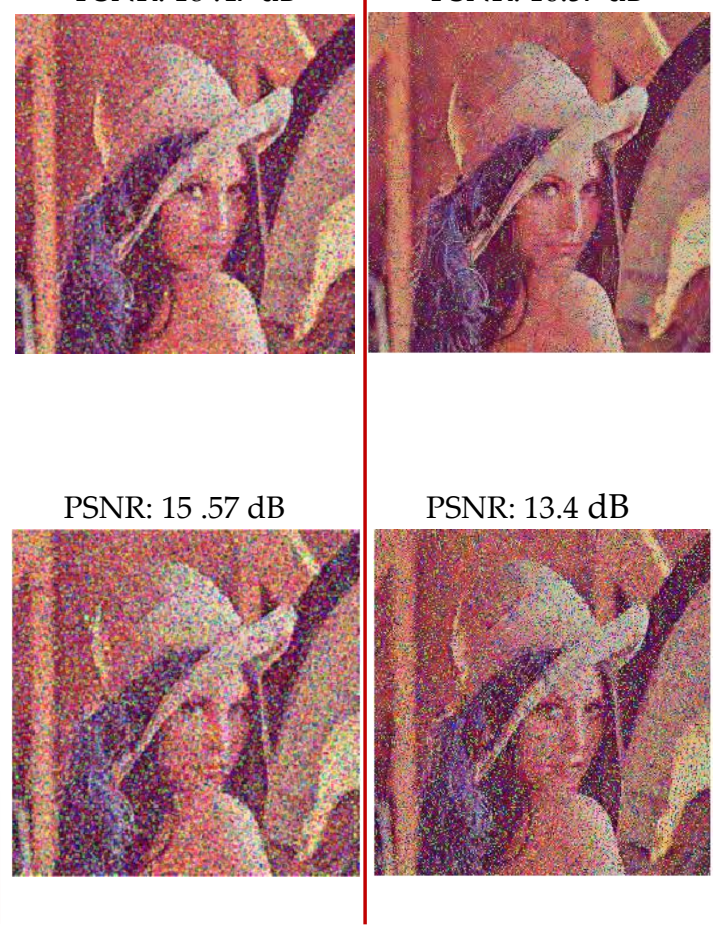

Figure 15. Restoration results of the salt and pepper noise-corrupted Lena image for different values of $\sigma$ (see column 1 located on the left side of the figure). The algorithms used, the proposed DCSR, wavelet denoising and GSR, are arranged in columns 2, 3 and 4 respectively. 


\subsection{Algorithm robustness}

In this sub-section, we will verify the robustness of the proposed algorithm. Applied on test images corrupted by additive white Gaussian noise, salt and pepper noise at different noise level.

Figures (17-20) below plot the values PSNR as a function of the number of iterations for gray Barbara and Cameraman images and color Lena image with various algorithms.

From these figures, it can be concluded that as the number of iterations increases, all PSNR curves increase monotonically and stabilize from the 10th iteration on wards. This shows that all these algorithms converge very quickly.

Nevertheless, the most robust algorithm should show a high PSNR. According to these figures, what ever the nature of the noise or the test image used, DCSR exhibits the highest PSNR, it is thus the most robust algorithm among the competing methods.

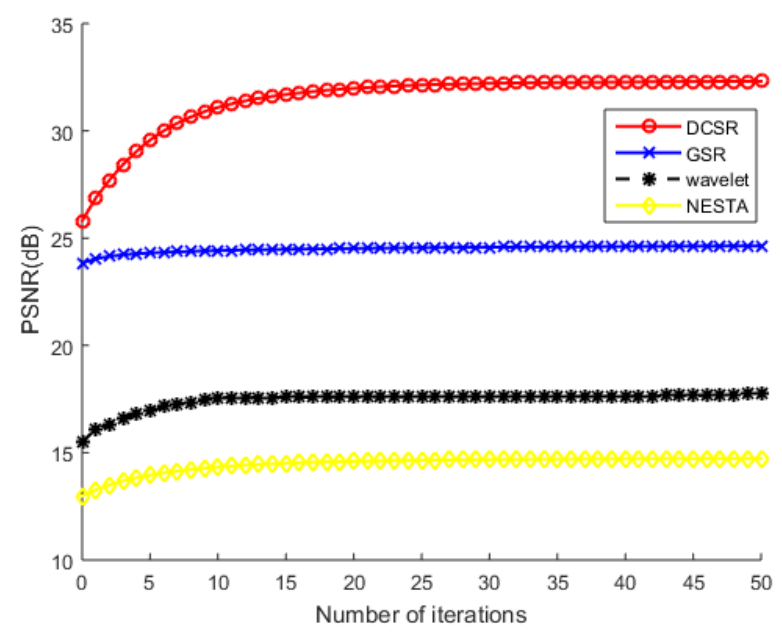

Figure 17. PSNR values of Barbara's grayscale image recovered by four competing methods as a function of the number of iterations. The test image is corrupted by AWGN.

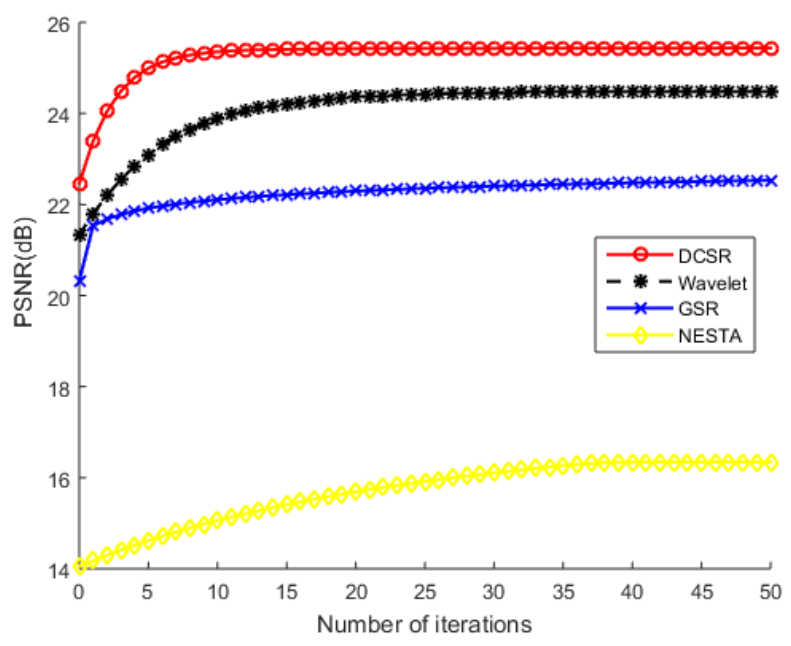

Figure 18. PSNR values of the Cameraman grayscale image recovered by four competing methods as a function of the number of iterations. The test image is corrupted by salt and pepper noise. 


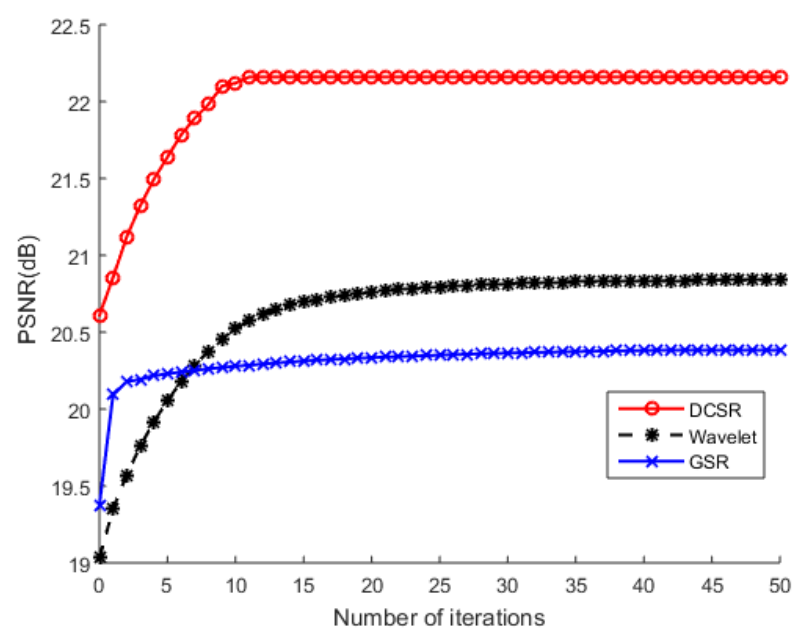

Figure 19. PSNR values of the recovered Lena color image by the competing methods vs. iterations number. The test image is corrupted by AWGN.

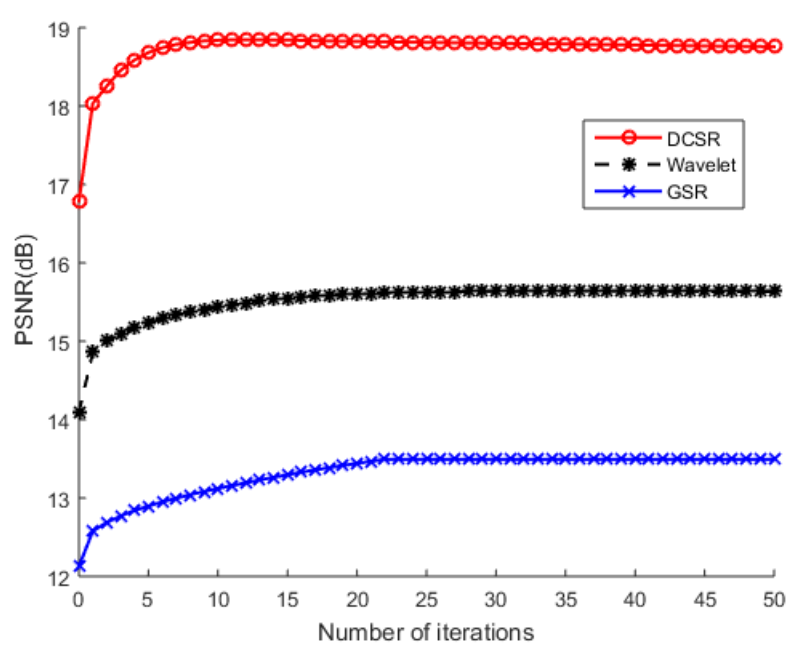

Figure 20. PSNR values of the recovered Lena color image by the competing methods vs. iterations number. The test image is corrupted by salt and pepper noise.

\subsection{Computational complexity}

In the following paragraph, we will estimate the computational complexity of the proposed DCSR algorithm. It is clear that the main complexity of the proposed algorithm comes from total variation TV and the high cost of the non-local self-similarities (NLS). Knowing that the computational complexity of TV is $O(N)$ [40], let us compute that of NLS: For an image $u$ of $N$ pixels, the average time to compute similar blocks for each reference block is $T_{s}$.

If $\sqrt{n} \times \sqrt{n}$ represents an overlapped blocks $u^{i}, i=1,2, \ldots, N$ and $(m-1)$ is the number of similar blocks denoted $S_{u^{i}}$, all blocks of $S_{u^{i}}$ are stacked in a matrix of size $(n \times m)$ with complexity $O\left(n \times m^{2}\right)$, hence a resulting complexity $O\left(N\left(n m^{2}+T_{s}\right)\right)$, just like the computational complexity of the group-based sparse representation GSR [41].

Therefore, we can conclude that the total computational complexity of our algorithm is $O\left(N\left(n m^{2}+T_{s}\right)+N\right)$.

It is interesting to compare the computational complexity of DCSR with competing methods :

The computational complexity of NESTA algorithm is $O\left(N+N \log _{2} N\right)$ [42] and that of wavelet denoising is $O\left(N \log _{2} N\right)$ [43].

Table 2 summarizes the computational complexity of the four algorithms used. 
The result of this comparison is as follows:

$O\left(N \log _{2} N\right)<O\left(N+N \log _{2} N\right)<O\left(N\left(n m^{2}+T_{s}\right)\right)<O\left(N\left(n m^{2}+T_{s}\right)+N\right)$

Relation (40) clearly shows that our proposed algorithm is more expensive in terms of computational complexity by an order $N$. Such an increase is not excessive in view of the good performance of this algorithm and the current computational means allowing real time processing.

Table 2. Computational complexity.

\begin{tabular}{cc}
\hline Algorithms & Computational complexity \\
\hline TV & $O(N)[40]$ \\
wavelet denoising & $O\left(N \log _{2} N\right)[43]$ \\
NESTA & $O\left(N+N \log _{2} N\right)[42]$ \\
GSR & $O\left(N\left(n m^{2}+T_{s}\right)\right)[41]$ \\
Our algorithm DCSR & $O\left(N\left(n m^{2}+T_{s}\right)+N\right)$ \\
\hline
\end{tabular}

\section{Conclusions}

In this paper, we proposed an original image denoising method based on compressed sensing that we called, denoising compressed sensing by regularizations terms (DCSR), by incorporating two regularization constraints: total variation and non-local selfsimilarity in the model. The optimization of this method is performed by the augmented Lagrangian which avoids the difficult problem of nonlinearity and non-differentiability of the regularization terms.

The effectiveness of our approach was validated using images corrupted by white Gaussian noise and impulsive salt and pepper noise.

Comparing DCSR in terms of PSNR and SSIM to state-of-the-art methods such as Nesterov's algorithm, group-based sparse representation and wavelet-based methods, it turns out that depending on the image texture and the type of noise corrupting the image, our method performs much better: we gain in PSNR at least 25\%, and in SSIM at least $11 \%$.

The price to pay is a slight increase in terms of computational complexity of order of image size, but this does not call into question the real time processing.

Due to the robustness and the speed of convergence of DCSR algorithm, its application is efficient in vital and sensitive domains such as medical imaging and remote sensing.

Our future contribution is a technological breakthrough consisting in introducing a layer of intelligence at the acquisition level aiming at automatically determining the image texture and its quality in terms of noise level, blur and shooting conditions (lighting, inpainting, registration, occlusion, low resolution, etc) [44-47] in order to automatically adjust the parameters necessary for an optimal use of the proposed DCSR algorithm.

Author Contributions: Software, investigation, formal analysis, writing, A.E.M.; methodology, validation and writing-review and editing A.O.; methodology, project administration and funding acquisition, M.S.M. All authors have read and agreed to the published version of the manuscript.

Funding: This research received no external funding.

Institutional Review Board Statement: Not applicable.

Data Availability Statement: Not applicable.

Conflicts of Interest: The authors declare no conflict of interest. 


\section{References}

1. Yang, Y.; Qin, X.; Wu, B. Fast and accurate compressed sensing model in magnetic resonance imaging with median filter and split Bregman method. IET Image Processing. 2019, 13, 1-8.

2. Labat, V.; Remenieras, J.; Matar, O.B.; Ouahabi, A.; Patat, F. Harmonic propagation of finite amplitude sound beams: Experimental determination of the nonlinearity parameter B/A. Ultrasonics. 2000, 38, 292-296.

3. He, J.; Zhou, Y.; Sun, G.; Xu, Y. Compressive multi-attribute data gathering using hankel matrix in wireless sensor networks. IEEE Communications Letters. 2019, 23, 2417-2421.

4. Haneche, H.; Ouahabi, A.; Boudraa, B. New mobile communication system design for Rayleigh environments based on compressed sensing-source coding. IET Commun. 2019, 13, 2375-2385.

5. Haneche, H.; Boudraa, B.; Ouahabi, A. A new way to enhance speech signal based on compressed sensing. Measurement. 2020, 151, 107-117.

6. Haneche, H.; Ouahabi, A.; Boudraa, B. Compressed sensing-speech coding scheme for mobile communications. Circuits Syst. Signal Process. 2021, 40, 5106-5126.

7. Li, H.; Li, S.; Li, Z.; Dai, Y.; Jin, T. Compressed sensing imaging with compensation of motion errors for MIMO Radar. Remote Sens. 2021, 13, 4909.

8. Andras, I.; Dolinský, P.; Michaeli, L.; Šaliga, J. A time domain reconstruction method of randomly sampled frequency sparse signal. Measurement 127. 2018, 68-77.

9. Mimouna, A.; Alouani, I.; Ben Khalifa, A.; El Hillali, Y.; Taleb-Ahmed, A.; Menhaj, A.; Ouahabi, A.; Ben Amara, N.E. OLIMP: A heterogeneous multimodal dataset for advanced environment perception. Electronics. 2020, 9, 560.

10. Ouahabi, A.; Depollier, C.; Simon, L.; Koume, D. Spectrum estimation from randomly sampled velocity data [LDV]. IEEE Trans.Instrum. Meas. 1998, 47, 1005-1012.

11. Ouahabi, A. A review of wavelet denoising in medical imaging. $8^{\text {th }}$ International Workshop on Systems, Signal processing and their applications (IEEE/WoSSPA), Algiers, Algeria, 12-15 May 2013; pp. 19-26.

12. Ahmed, S.S.; Messali, Z.; Ouahabi, A.; Trepout, S.; Messaoudi, C.; Marco, S. Nonparametric denoising methods based on contourlet transform with sharp frequency localization: Application to low exposure time electron microscopy images. Entropy. 2015, 17, 3461-3478.

13. Ouahabi, A. Signal and Image Multiresolution Analysis; ISTE-Wiley: London, UK; Hoboken, NJ, USA, 2013.

14. Femmam, S.; M'Sirdi, N.K.; Ouahabi, A. Perception and characterization of materials using signal processing techniques. IEEE Trans. Instrum. Meas. 2001, 50, 1203-1211.

15. Chambolle, A. An algorithm for total variation minimization and applications. J. Math. Imaging Vis. 2004, 20, 89-97.

16. Beck, A.; Teboulle, M. Fast Gradient-based algorithms for constrained total variation image denoising and deblurring problems. IEEE Trans. Image Process.2009, 18, 11, 2419-2434.

17. Zhang, H.; Liu, L.; He, W.; Zhang, L. Hyperspectral image denoising with total variation regularization and nonlocal low-rank tensor decomposition. IEEE Transactions on Geoscience and Remote Sensing. 2020, 58, 5, 3071-3084.

18. Wang, M.; Wang, Q.; Chanussot, J. Tensor low-rank constraint and lo total variation for hyperspectral image mixed noise removal. IEEE Journal of Selected Topics in Signal Processing. 2021, 15, 3, 718-733.

19. Candes, E.J.; Romberg, J.K.; Tao, T. Robust uncertainty principles: exact signal reconstruction from highly incomplete frequency information. IEEE Trans. Inform. 2006, 52, 489-509.

20. Eldar, Y.C.; Kutyniok, G. Compressed Sensing: Theory and Applications. Cambridge University Press, 2012.

21. Tropp, J. A. Greed is good: algorithmic results for sparse approximation. IEEE Trans. Inf. Theory. 2004, 50, $2231-2242$.

22. Donoho, D.L.; Malekib, A.; Montanaria, A. Message-passing algorithms for compressed sensing. Proc. Natl. Acad. Sci. 2009, 106, 45, 18914-18919.

23. Metzler, C.A.; Maleki, A.; Baraniuk, R.G. From denoising to compressed sensing. IEEE Trans. Inf. Theory. 2016, 62, 5117-5144.

24. Hestenes, M.R., Multiplier and gradient methods. J. Optim. TheoryAppl. 1969, 4, 303-320.

25. Nocedal, J.; Wright, S. J. Numerical Optimization; Springer, 2006.

26. Blomgren, P; Chan, T.F. Color tv: total variation methods for restoration of vector-valued images. IEEE Trans. Image Process. 1998, 7, 304-309.

27. Mallat, S. A Wavelet Tour of Signal Processing: The Sparse Way; Academic Press, 2008.

28. Buades, A.; Coll, B.; Morel, J. M. Nonlocal image and movie denoising. Int. J.Comput. Vision. 2008, 76, 123-139.

29. Chen, S.S.; Donoho, D.L.; Saunders, M.A. Atomic decomposition by basis pursuit. SIAM. J. Sci Comput. 1998, $20,33-61$.

30. Dabov, K.A.; Katkovnik, V.; Egiazarian, K. Image denoising by sparse 3-d transform-domain collaborative filtering. IEEE Trans Image Process. 2007, 16, 2080-2095.

31. Rudin, L.I.; Osher, S.; Fatemi, E. Non linear total variation based noise removal algorithms. Physica D. 1992, 60, 1-4, $259-268$.

32. Buades, A.; Coll, B.; Morel, J.M. A non-local algorithm for image denoising. In Proc. IEEE Conf. Comput. Vis. Pattern Recognit. 2005 ; pp. 60-65.

33. Ouahabi, A. Multifractal Analysis for Texture Characterization: A New Approach Based on DWT. In Proceedings of the 10th International Conference on Information Science, Signal Processing and their Applications (ISSPA 2010), Kuala Lumpur, Malaysia, 10-13 May 2010; pp. 698-703.

34. Djeddi, M.; Ouahabi, A.; Batatia, H.; Basarab, A.; Kouam, D. Discrete wavelet for multifractal texture classification: application to medical ultra sound imaging. IEEE International Conference on Image Processing, Hong Kong, 2010; pp. 637-640. 
35. Donoho, D.L; Johnstone, I. M.; Kerkyacharian, G.; Picard, D. Wavelet shrinkage. Asymptopia. J. Roy. Statist. Soc. B. 1995, 57, 301-337.

36. Donoho, D.L; Johnstone, I.M. Ideal spatial adaptation by wavelet shrinkage. Biometrika. 1994, 81, 425-455.

37. Li, C.; Yin, W.; Jiang, H.; Zhang, Y. An efficient augmented Lagrangian method with applications to total variation minimization. Comput. Optim. Appl. 2013, 56, 507-530.

38. Ferroukhi, M.; Ouahabi, A.; Attari, M.; Habchi, Y.; Taleb-Ahmed, A. Medical video coding based on 2nd-generation wavelets: Performance Evaluation. Electronics. 2019, 8, 88.

39. Wang, Z.; Bovik, A.C.; Sheikh, H.R.; Simoncelli, E.P. Image quality assessment: From error visibility to structural similarity. IEEE Trans. Image Processing. 2004, 13, 4, 600-612.

40. Daei, S.; Haddadi, F.; Amini, A. Sample complexity of total variation minimization. IEEE Signal Processing Letters. 2018, 25, 8, 1151-1155.

41. Zhang, J.; Zhao, D.; Gao, W. Group-based sparse representation for image restoration. IEEE Transactions on Image Processing. 2014, 23, 8, 3336-3351.

42. Becker, S.; Bobin, J.; Candes, E.J. NESTA: A fast and accurate first-order method for sparse recovery. SIAM journal on imaging sciences. 2011, 4, 1, 1-39.

43. Srivastava, M.; Anderson, C.L; Freed, J.H. A New wavelet denoising method for selecting decomposition levels and noise thresholds. IEEE Access. 2016, 4, 1, 3862-3877.

44. Ouahabi, A.; Taleb-Ahmed, A. Deep learning for real-time semantic segmentation: Application in ultrasound imaging. Pattern Recognit. Lett. 2021, 144, 27-34.

45. Adjabi, I.; Ouahabi, A.; Benzaoui, A.; Taleb-Ahmed, A. Past, present, and future of face recognition: A Review. Electronics. 2020, 9, 1188.

46. Adjabi, I.; Ouahabi, A.; Benzaoui, A.; Jacques, S. Multi-block color-binarized statistical images for single-sample face recognition. Sensors. 2021, 21, 728 .

47. Khaldi, Y.; Benzaoui, A.; Ouahabi, A.; Jacques, S.; Taleb-Ahmed, A. Ear recognition based on deep unsupervised active learning. IEEE Sens. J. 2021, 21, 18, 20704-20713. 